Revue internationale P.M.E.

Économie et gestion de la petite et moyenne entreprise

\title{
Comprendre l'ancrage territorial d'une bio-industrie : le cas de la grappe biopharmaceutique de la région de Montréal (Canada)
}

\author{
Anne-Laure Saives et Robert H. Desmarteau
}

Volume 18, numéro 2, 2005

URI : https://id.erudit.org/iderudit/1008476ar

DOI : https://doi.org/10.7202/1008476ar

Aller au sommaire du numéro

Éditeur(s)

Presses de l’Université du Québec

ISSN

0776-5436 (imprimé)

1918-9699 (numérique)

Découvrir la revue

Citer cet article

Saives, A.-L. \& Desmarteau, R. H. (2005). Comprendre l'ancrage territorial d'une bio-industrie : le cas de la grappe biopharmaceutique de la région de Montréal (Canada). Revue internationale P.M.E., 18(2), 75-108.

https://doi.org/10.7202/1008476ar
Résumé de l'article

La grappe des activités biopharmaceutiques de la région de Montréal est aujourd'hui reconnue internationalement. L'objet de cette contribution est de décrire l'ancrage territorial de ces activités à partir de quatre dynamiques conçues comme créatrices de valeur dans une grappe industrielle : la capitalisation de savoirs, la dynamique d'optimisation des coûts, la double logique de concurrence et coopération et la dynamique de gouvernance. Une enquête menée auprès de 45 entreprises révèle que ces dynamiques reposent sur cinq moteurs stratégiques activés différemment par quatre types d'entreprises interreliées (entreprises pharmaceutiques, entre prises de recherche clinique à contrat [CRO], entreprises de biotechnologies [EDB] " maillées » et en démarrage) dont les ancrages organisationnels complémentaires caractérisent la « construction territoriale " montréalaise. 


\title{
Comprendre l'ancrage territorial d'une bio-industrie : le cas de la grappe biopharmaceutique de la région de Montréal (Canada)
}

\author{
Anne-Laure SAIVES \\ Robert H. DESMARTEAU \\ École des sciences de la gestion \\ Université du Québec à Montréal
}

MOTS CLÉS

Grappe industrielle - Dynamiques territoriales
Système industriel de création de valeur - Ressources
Bio-industrie - Biotechnologie - Biopharmaceutique

LES AUTEURS

ANNE-LAURE SAIVES est professeure de management stratégique et chercheure au sein du Groupe de recherche sur le management et la transformation des entreprises de biotechnologie (MATEB) à l'École des sciences de la gestion de l'Université du Québec à Montréal. Ses travaux portent sur l'analyse de la compétitivité à base territoriale des firmes, en particulier sur l'analyse des stratégies d'ancrage des firmes de biotechnologies et leurs modèles d'affaires ainsi que sur les dimensions stratégiques des dynamiques territoriales des grappes bio-industrielles. Adresse: École des sciences de la gestion (ESG), Université du Québec à Montréal, 315, rue Sainte-Catherine Est, Montréal, Québec, H2X 3X2. Courriel <saives. anne-laure@uqam.ca>.

ROBERT H. DESMARTEAU est professeur agrégé de stratégie organisationnelle au Département de stratégie des affaires de l'Université du Québec à Montréal. En 1997, il a obtenu un doctorat en stratégie radicale de l'Université du Québec à Montréal après avoir œuvré pendant 13 ans dans la gestion hospitalière. Ses travaux de recherche portent sur la création de valeur économique à l'ère des réseaux, la synergie des capitaux économique et social et la modélisation des dynamiques stratégiques dans les grappes de compétences des sciences de la vie. Adresse: École des sciences de la gestion (ESG), Université du Québec à Montréal, 315, rue Sainte-Catherine Est, Montréal, Québec, H2X 3X2. Courriel $<$ desmarteau.robert_h@uqam.ca>.

Remerciements

Nous tenons ici à remercier Frédéric Corolleur, maître de conférences de l'Université PierreMendès-France, Unité mixte de recherche GAEL et Jacques Perrat, directeur scientifique à l'Association pour le développement des études économiques et sociales (ADEES) en Rhône-Alpes, pour leurs commentaires constructifs à la lecture d'une version précédente de cet article, ainsi que Xavier Olleros, professeur à l'École des sciences de la gestion de I'Université du Québec à Montréal pour sa traduction espagnole du résumé. 


\section{RÉSUMÉ}

La grappe des activités biopharmaceutiques de la région de Montréal est aujourd'hui reconnue internationalement. L'objet de cette contribution est de décrire l'ancrage territorial de ces activités à partir de quatre dynamiques conçues comme créatrices de valeur dans une grappe industrielle: la capitalisation de savoirs, la dynamique d'optimisation des coûts, la double logique de concurrence et coopération et la dynamique de gouvernance. Une enquête menée auprès de 45 entreprises révèle que ces dynamiques reposent sur cinq moteurs stratégiques activés différemment par quatre types d'entreprises interreliées (entreprises pharmaceutiques, entreprises de recherche clinique à contrat [CRO], entreprises de biotechnologies $[E D B]$ «maillées » et en démarrage) dont les ancrages organisationnels complémentaires caractérisent la «construction territoriale» montréalaise.

\section{ABSTRACT}

The Montreal biopharmaceutical cluster is now internationally well-known. To describe its organization, four strategic territorial dynamics are conceptualized and analyzed: the knowledge capitalization, costs optimization, competitioncooperation and governance dynamics. A survey of 45 firms reveals that those territorial dynamics result from five strategic drivers activated by four types of enterprises (pharmaceutical enterprises, contract research organization, "networked" biotechnology firms and "start-up" biotechnology firms) whose organizational anchorages take part into the performing Montreal biopharmaceutical territorial construction.

\section{RESUMEN}

La aglomeración de actividades bio-farmacéuticas de la región de Montreal es una realidad apreciada internacionalmente. El objetivo de este trabajo es describir la localización territorial de estas actividades a partir de cuatro dinámicas creadoras de valor en una aglomeración industrial: la capitalización del saber, la dinámica de optimización de costes, la doble lógica de competencia y cooperación y la dinámica de governancia. Una encuesta realizada en 45 empresas revela que éstas dinámicas reposan sobre cinco motores estratégicos activados diferentemente por cuatro tipos de empresas entrelazadas - empresas farmacéuticas, empresas de investigación clínica bajo contrato, empresas de biotecnología en red y empresas de biotecnología en despegue - cuyos entramados organizacionales complementarios caracterizan la construcción territorial de la región de Montreal.

\section{ZUSAMMENFASSUNG}

Die Aktivitäten des bio-pharmazeutischen Clusters der Region Montreal sind heute international anerkannt. Gegenstand dieser Publikation ist der Beschrieb der regionalen Verankerung dieser Aktivitäten anhand der vier Antriebskräfte, die als konstituierend für ein Industrie-Cluster gelten: die Kapitalisierung von Knowhow, die dynamische Kostenoptimierung, das Zusammenspiel von Konkurrenz 
und Kooperation und die dynamische Unternehmensführung. Eine Untersuchung bei 45 Unternehmen brachte hervor, dass die Dynamik auf fünf verschiedenen strategischen Motoren basieren, die unterschiedlich durch vier miteinander verbundene Typen von Unternehmen aktiviert werden. Zum einen durch pharmazeutische Unternehmen und Unternehmen klinischer Vertragsforschung, zum anderen durch bestandene und durch neu gegründete Biotechnologieunternehmen. Diese Unternehmen charakterisieren den Aufbau des Clusters in der Region Montreal.

\section{Introduction}

Les entreprises des bio-industries ou encore des systèmes bio-industriels de création de valeur résultent d'un processus d'industrialisation des innovations biotechnologiques ${ }^{1}$, elles-mêmes issues de la transformation des inventions fondées sur les biosciences (Saives, Desmarteau et Seni, 2003). Sous l'impulsion de stratégies gouvernementales successives ciblées visant, entre autres, à promouvoir le développement des sciences de la vie et des biotechnologies localement (Picard, 1986), la région de Montréal (Canada), aujourd'hui internationalement reconnue comme un «cluster biotech», concentre une grande majorité des entreprises des systèmes bio-industriels de la santé humaine, de la nutrition, de l'agriculture et de l'environnement du Canada (IPSÉ, 2002). La variété des types d'entreprises et d'institutions y est beaucoup plus élevée qu'ailleurs, en particulier dans le secteur prépondérant et compétitif de la santé humaine (Desmarteau, Niosi et Saives, 2002). Dès le début du siècle dernier, l'émulation résultant de la présence de deux réseaux universitaires distincts (francophone et anglophone) et de services de santé adaptés (multiethniques), a significativement contribué à l'avènement de Montréal comme un centre de recherche de calibre international dans les sciences de la vie et de la santé et a incité plusieurs entreprises de fabrication de produits pharmaceutiques à venir s'y installer. À Montréal, en dehors des secteurs de la nutrition, de l'agriculture et de l'environnement, coexistent près de 100 entreprises dédiées aux biotechnologies en santé humaine, des entreprises multinationales de recherche pharmaceutique, des entreprises pharmaceutiques produisant des médicaments génériques, des organisations de recherche clinique à contrat, en plus de sociétés de chimie combinatoire, plusieurs hôpitaux de recherche, quatre universités de recherche anglophones et francophones (Germain et Rose, 2000) et le plus grand laboratoire public (fédéral) de recherche en biotechnologie (l'Institut de

1. Voici comment l'OCDE (2002) définit la biotechnologie: «The application of $S \& T$ to living organisms as well as parts, products and models thereof, to alter living or non-living materials for the production of knowledge, goods and services. »

Revue internationale P.M.E., vol. 18, nº 2, 2005

(c) 2005 - Presses de l'Université du Québec

Édifice Le Delta I, 2875, boul. Laurier, bureau 450, Sainte-Foy, Québec G1V 2M2 • Tél.: (418) 657-4399 - www.puq.ca

Tiré de: Revue internationale P.M.E., vol. 18, $\mathrm{n}^{\circ} 2$, sous la direction de Louis Raymond • PME1802N

Tous droits de reproduction, de traduction et d'adaptation réservés 
recherche en biotechnologie du Conseil national des recherches du Canada) ${ }^{2}$. Ce constat d'intérêt façonne l'objet de cet article et motive la formulation de la question de recherche suivante: quelle construction territoriale participe de l'ancrage de la grappe biopharmaceutique dans la région de Montréal?

Pour y répondre, nous procéderons en trois temps en présentant d'abord un cadre théorique d'instrumentation des dimensions des quatre dynamiques territoriales d'une grappe industrielle de compétences, en expliquant ensuite la méthode adoptée pour caractériser le terrain québécois et en analysant enfin les résultats de nos enquêtes et le comportement territorial - à savoir l'activation différenciée de moteurs stratégiques locaux spécifiques - des différents types d'entreprises interreliées issus de l'analyse multifactorielle dans la région de Montréal.

\section{Les quatre dynamiques territoriales d'une grappe industrielle de compétences}

Pour modéliser de façon systémique les dynamiques régionales des entreprises des bio-industries au Québec, nous utilisons la notion de grappe (cluster) introduite par Marshall dès le début du Xx $x^{\mathrm{e}}$ siècle au Royaume-Uni (avec l'idée d'effets externes à la firme ${ }^{3}$ ) et développée de façon contemporaine dans le concept de grappe industrielle, notamment dans les travaux de Porter (1993, 1998), Fujita, Krugman et Venables (1999) ou Beccattini (1998). En effet, au pourquoi des concentrations économiques de firmes expliquées par les économies d'agglomération (Krugman, 1991), les économies d'urbanisation, etc., l'approche organisationnelle des économies d'agglomération, notamment par le biais des grappes de compétences ${ }^{4}$, permet d'amorcer une réflexion sur le comment de la création

2. «Montréal is the primary health and life-sciences research center in Canada [...]. The city has a large and closely integrated complex of university hospitals and specialized research centers, some of which are among the world leaders in several fields of clinical research and practice [...] This research network's capacity to produce leading-edge specialists is enhanced by the unique access to both French-and English-language international networks of medical expertise» (Germain et Rose, 2000, p. 139; c'est nous qui soulignons). $C f$. également Cortright et Mayer (2002).

3. Marshall a ainsi insisté sur trois types d'économies externes qui peuvent orienter les localisations industrielles: la présence d'externalités de savoir entre compétiteurs et la demande industrielle qui impulse, d'une part, la création d'un bassin de main-d'œuvre qualifiée et, d'autre part, la création d'un bassin de fournisseurs spécialisés.

4. Grappes ou clusters qui peuvent prendre différentes formes dont celle des districts technologiques (DT), district industriel (DI), etc. (Porter, 1998).

Revue internationale P.M.E., vol. 18, nº 2, 2005

(C) 2005 - Presses de l'Université du Québec

Édifice Le Delta I, 2875, boul. Laurier, bureau 450, Sainte-Foy, Québec G1V 2M2 • Tél.: (418) 657-4399 - www.puq.ca

Tiré de: Revue internationale P.M.E., vol. 18, $\mathrm{n}^{\circ} 2$, sous la direction de Louis Raymond PME1802N

Tous droits de reproduction, de traduction et d'adaptation réservés 
de valeur par l'analyse de la boîte noire des organisations et de leurs interrelations dans une chaîne de valeur (Roelandt et den Hertog, 1999) ou encore dans un système de valeur (Parolini, 1999). De plus, alors que la plupart des études relevées dans la littérature économique portent sur la population exclusive des entreprises d'innovation biotechnologique ${ }^{5}$, nous examinerons l'ensemble du système des entreprises créatrices de valeur dans le domaine de l'industrialisation des biotechnologies de la santé humaine. Aussi, nous retenons la proposition de Porter (1998) où la «grappe» s'associe à «des agglomérations géographiques de firmes interreliées, de fournisseurs spécialisés de biens et de services et d'institutions associées dans un champ donné où l'on dégage des attributs communs et des complémentarités dans une double logique de concurrence et de coopération ${ }^{6}$. Cette définition s'arrime aussi aux nombreuses réflexions menées sur les notions de milieu local (GREMI, 1993) et de territoire par Aydalot (1985), Perrin (1991) ou encore Rallet et Torre (1995), pour lesquels le territoire s'associe à une «matrice» de compétences et d'expertises. Comme l'écrit Sierra (1997, p. 10), il s'agit de ne plus traiter le territoire comme un «espace neutre, un réceptacle de technologies définies de façon exogène, mais comme un "vecteur" indissociable de leur émergence et de leur développement », idiosyncratique et historiquement construit (Colletis, Gilly et Pecqueur, 2001; Bartholomey, 1997). Didactiquement, toutes ces réflexions convergent pour reconnaître les grappes industrielles et les milieux locaux ou encore les territoires, comme de véritables tremplins stratégiques («influençant la compétition» selon les termes de Porter [1998, p. 9]) qui accélèrent la performance des entreprises qui y sont «ancrées» (Zimmermann, 2001), plus ou moins durablement.

Dans la mesure où, néanmoins, la dynamique territoriale influe différemment selon l'hétérogénéité des firmes (Shaver et Flyer, 2000), dans le cas de la grappe industrielle de la santé humaine, nous intégrerons de façon holistique les compétences clés des divers types d'entreprises biopharmaceutiques et la séquence de leurs activités interreliées. Dans ces secteurs qui visent essentiellement la production de médicaments chimiques ou biologiques, nous nous limiterons à la succession des trois phases principales (figure 1) des activités industrielles (bio)pharmaceutiques, depuis la recherche fondamentale jusqu'à la mise en marché (production et commercialisation) en passant par l'étape intermédiaire de

5. Cf., par exemple, Pisano (1991), Powell (1998), DeCarolis et Deeds (1999), et Stuart et Sorenson (2003).

6. Ou encore un cluster peut être défini comme un système de firmes interconnectées et d'institutions dont la valeur comme un tout est supérieure à la somme de ses parties (Porter, 1998, p. 13).

Revue internationale P.M.E., vol. 18, n 2, 2005

(C) 2005 - Presses de l'Université du Québec

Édifice Le Delta I, 2875, boul. Laurier, bureau 450, Sainte-Foy, Québec G1V 2M2 • Tél.: (418) 657-4399 - www.puq.ca

Tiré de: Revue internationale P.M.E., vol. 18, n², sous la direction de Louis Raymond • PME1802N

Tous droits de reproduction, de traduction et d'adaptation réservés 
validation des fonctionnalités des produits finals ou des technologies, c'est-à-dire la recherche clinique (design de recherche préclinique, recherche préclinique puis recherche clinique; Depret et Hamdouch, 2000; Pisano, 1994), en excluant a priori de l'analyse les activités de services (agents de brevets, fournisseurs de produits de laboratoire, etc.).

Enfin, pour comprendre la finalité du système d'éléments interreliés que constitue une grappe industrielle de compétences, il convient de réfléchir à son association au principe de la création de valeur (Stewart, 1991; Christensen, 1997; Parolini, 1999; OCDE, 1999). Joffre et Koenig (1992, p. 149-161) sont parmi les premiers à souligner que «les aires-systèmes » comme représentations de l'univers stratégique, à la fois territoire géographique et système social en symbiose avec l'organisation, contribuent de façon cruciale à la création de valeur et à la compétitivité des entreprises qu'elles accueillent en permettant l'accès 1) à des actifs spécifiques locaux que des acteurs extérieurs ne peuvent mobiliser avec la même efficience; 2) à une réduction des coûts de transaction ${ }^{7}$ dans l'univers moins incertain de relations industrielles plus conventionnelles (fondées sur la confiance) que contractuelles et, enfin, 3) à un milieu favorable au développement de compétences spécialisées et spécifiques, par le jeu de la stimulation de processus d'apprentissages collectifs (GREMI, 1993; Julien et Marchesnay, 1996).

Pour résumer les déterminants territoriaux de la création de valeur dans une grappe industrielle de compétences, à partir de la littérature des économistes industriels et régionaux évoquée précédemment ${ }^{8}$, tout comme des études récentes publiées par l'OCDE (2001a, 2001b, 1999), nous proposons de caractériser les quatre dynamiques spécifiques locales porteuses de valeur et d'avantages compétitifs dans le cas de secteurs industriels high-tech innovants, c'est-à-dire 1) la capitalisation de savoirs, 2) l'optimisation des coûts, 3) la double logique de concurrence et coopération et 4) la gouvernance organisationnelle. Ces quatre dynamiques sont représentées à la figure 1 et explicitées ci-après.

7. Les coûts de transaction sont les coûts «de friction », c'est-à-dire d'information et de négociation qui accompagnent l'établissement d'un contrat (Williamson, 1985, p. 19-22).

8. Ainsi que, récemment, Amin et Cohendet (2000), Colletis, Gilly et Pecqueur (2001) et Lacetera (2001).

9. Cette dynamique permet d'exploiter plus pleinement la portée de la pensée et de l'exercice de la stratégie organisationnelle à l'échelle de la firme (Lacetera, 2001), du système industriel dont elle fait partie ou encore de son territoire d'appartenance (Colletis, Gilly et Pecqueur, 2001).

Revue internationale P.M.E., vol. 18, nº 2, 2005

(C) 2005 - Presses de l'Université du Québec

Édifice Le Delta I, 2875, boul. Laurier, bureau 450, Sainte-Foy, Québec G1V 2M2 • Tél.: (418) 657-4399 - www.puq.ca

Tiré de: Revue internationale P.M.E., vol. 18, $\mathrm{n}^{\circ} 2$, sous la direction de Louis Raymond PME1802N

Tous droits de reproduction, de traduction et d'adaptation réservés 


\subsection{La dynamique de la capitalisation de savoirs}

La capitalisation de savoirs se concrétise par la reconnaissance locale et globale de compétences scientifiques et technologiques distinctives qui donnent à l'entreprise et à la grappe de compétences à laquelle elle appartient un avantage compétitif. La capitalisation de savoirs, c'est la construction d'un double capital cognitif individuel et organisationnel autour de systèmes de connaissances de pointe et d'informations «riches» (Julien et Marchesnay, 1996, p. 98). (Voir le tableau récapitulatif 1.$)$

Le capital humain se développe à partir de deux moteurs stratégiques: la création et la diffusion de savoirs. La création de savoirs profite des fertilisations croisées essentiellement entre entreprises et universités que rendent possibles la pluridisciplinarité des compétences scientifiques et technologiques locales et la multi-institutionnalité (Bartholomey, 1997; Powell, 1998). Le savoir se diffuse essentiellement par des processus de transfert comme la publication (légitimation des savoirs scientifiques et technologiques produits), la formation, la communication-socialisation (Corno, Reinmoeller et Nonaka, 1999) et la mobilité de la main-d'œuvre (Almeida et Kogut, 1999). Le capital organisationnel résulte de l'institutionnalisation du savoir dans des groupes de recherche ou des réseaux sociaux de partenariats plus ou moins locaux (Pisano, 1991; Liebeskind et al., 1996; Powell, 1998; DeCarolis et Deeds, 1999; Baum, Calabrese et Silverman, 2000), dans la spécialisation de ces groupes autour d'un centre de gravité reconnu (focus), stimulé par la proximité et l'engagement de figures de proue (stars scientists, inventeurs-entrepreneurs). Ce capital organisationnel est inscrit dans les routines de ces groupes et pérennisé par le biais d'apprentissages collectifs par une main-d'œuvre hautement spécialisée. Cette institutionnalisation du savoir dépend du système tout en lui conférant une capacité d'absorption (Cohen et Levinthal, 1990 ; Cockburn et Henderson, 1998) d'actifs maillés complémentaires ou encore une capacité d'apprentissage dynamique (Ingelgard et al., 2002) difficilement imitable (Grant, 1996), porteuse d'interactions potentiellement fertiles, essentielle à la flexibilité et à la compétitivité du processus d'innovation.

Dans les secteurs d'activité des bio-industries de la santé humaine, la capitalisation de savoirs, c'est la construction d'organisations, voire d'institutions qui reposent sur des plates-formes scientifiques et technologiques distinctives, fondées sur une masse critique de connaissances complexes et spécifiques enregistrées, par exemple, dans des brevets d'invention (un stock de savoirs) reconnus comme source de valeur (Lerner, 1994). Scruter la dynamique de capitalisation de savoirs dans une grappe de compétences, c'est donc examiner les liens porteurs de création, de diffusion et d'institutionnalisation de savoirs à l'intérieur du système industriel et avec les acteurs de son environnement technologique.

Revue internationale P.M.E., vol. 18, n 2, 2005

(C) 2005 - Presses de l'Université du Québec

Édifice Le Delta I, 2875, boul. Laurier, bureau 450, Sainte-Foy, Québec G1V 2M2 • Tél.: (418) 657-4399 - www.puq.ca

Tiré de: Revue internationale P.M.E., vol. 18, n² 2, sous la direction de Louis Raymond • PME1802N

Tous droits de reproduction, de traduction et d'adaptation réservés 


\subsection{La dynamique d'optimisation des coûts}

Pour mettre en œuvre ses processus clés de création de valeur au sein d'une grappe industrielle de compétences, l'entreprise est tenue d'activer ${ }^{10}$ de façon efficiente des ressources à la fois humaines, financières, technologiques, matérielles, informationnelles, organisationnelles, voire légales ${ }^{11}$ (Saives, 2002). L'optimisation des coûts (tableau 1) résulte de l'expression de plusieurs facteurs liés à la concentration géographique de la grappe et à l'importance de sa masse critique permettant des économies d'agglomération, d'échelle et d'envergure. Le premier facteur est l'accessibilité à un bassin de main-d'œuvre qualifiée résultant de la concentration locale de l'activité économique mais qui peut défavorablement évoluer vers une concurrence néfaste en cas de congestion (Stuart et Sorenson, 2003). Le deuxième facteur s'associe à la présence d'une riche infrastructure publique et d'un secteur tertiaire développé pour aider ou soutenir les acteurs d'une grappe de compétences dans l'atteinte de leur performance (Bartholomey, 1997). Un troisième facteur d'optimisation des coûts prend son origine dans les déterminants environnementaux politiques de la gouvernance. Les systèmes politiques de gouvernance territoriale ou encore les systèmes de surveillance ou d'incitation environnementaux comprennent, entre autres, les cadres juridiques (accessibilité de la propriété intellectuelle), financiers (programmes publics de financement de la recherche fondamentale et appliquée, accessibilité des capitaux de risque publics ou privés; Feldman, 2001; Bartholomey, 1997) et fiscaux dont les crédits spécifiques obtenus par les entreprises effectuant de la recherche et développement sont un exemple. Le quatrième facteur regroupe toutes les formes de liens entre entreprises autonomes pour réduire leurs frais d'exploitation et ainsi diminuer les coûts de transaction. C'est en ce sens que les proximités géographique, économique et organisationnelle (Sierra, 1997) participent de l'accessibilité de ces ressources et compétences.

\subsection{La double logique de concurrence et de coopération}

Reconnue de façon générale comme un attribut porteur de valeur dans les grappes industrielles de compétences, la double logique de concurrence et de coopération se construit par des gestes de compétition et de partage entre acteurs à la

10. $C f$. Dierickx et Cool (1989) pour la distinction entre stock et flux de ressources.

11. Cette classification des ressources est inspirée de Hofer et Schendel (1978), Penrose (1959) et Barney (1991). Les «ressources légales» ajoutées renforcent les ressources financières (subventions, protection) et organisationnelles (lois, règlements). Précisons également que le terme générique «ressources» englobe ici les différentes catégories des ressources, des compétences et des capabilités (capacités dynamiques), distinguées par les théoriciens de la ressource.

Revue internationale P.M.E., vol. 18, nº 2, 2005

(C) 2005 - Presses de l'Université du Québec

Édifice Le Delta I, 2875, boul. Laurier, bureau 450, Sainte-Foy, Québec G1V 2M2 • Tél.: (418) 657-4399 - www.puq.ca

Tiré de: Revue internationale P.M.E., vol. 18, $\mathrm{n}^{\circ} 2$, sous la direction de Louis Raymond - PME1802N

Tous droits de reproduction, de traduction et d'adaptation réservés 
fois différents et complémentaires tout au long de la chaîne de valeur (Roelandt et Den Hertog, 1999). Dans les entreprises dédiées de biotechnologies (EDB), la participation à un réseau à travers des coopérations, des collaborations ou des alliances se révèle déterminante pour la croissance et le développement des entreprises (Powell, Koput et Smith-Doerr, 1996; Baum, Calabrese et Silverman, 2000; Catherine et Corolleur, 2003; Catherine, Corolleur et Coronini, 2003) et pour l'exploitation et la perfection des compétences distinctives d'une entreprise (Pisano, 1994; Powell, 1998) par la facilitation de l'accès à des compétences et des connaissances ou leur partage (Cohen et Levinthal, 1990; Ingelgard et al., 2002; Salman et Saives, 2005). Cette notion de réseau reprend, entre autres, les enseignements de Coleman (1988), Bourdieu (1986) et Putnam (1993) sur l'avantage concurrentiel de la présence d'un tissu social porteur de capital social où les individus partagent des normes, des valeurs et des compréhensions qui facilitent leur coopération (OCDE, 2001c). Le tableau 1 résume les fondements des logiques conjuguées de la coopération et de la concurrence. En effet, la coopération repose sur la recherche de synergies et sur des besoins de complémentarités (Arora et Gambardella, 1990; Surlemont, 2000) alors que la concurrence résulte de la volonté de défendre des compétences clés malgré la similarité des ressources, compétences ou encore des produits et/ou services des compétiteurs. Dans une grappe industrielle de compétences, la dynamique de coopération et de compétition façonne l'émulation ou encore le dépassement des acteurs en alimentant des capacités d'interaction et d'apprentissage variables au cours du temps (Oliver, 2001). La coopération entre acteurs d'une grappe industrielle de compétences peut se réaliser tant horizontalement dans des gestes qui miment les associations communautaires (Desmarteau, 2000) que verticalement pour l'obtention de complémentarités.

\subsection{La dynamique de la gouvernance}

Dans sa plus simple expression, la gouvernance représente l'exercice du pouvoir ultime par un corps constituant sur une entité organisationnelle. Dans une société à capital actions, le corps constituant s'incarne dans les membres du conseil d'administration à qui l'on confère des droits de surveillance, jugement et décision sur l'ensemble de la performance de l'entreprise, dont principalement ses capacités d'intégrer des compétences distinctives, de s'adapter à l'environnement et de créer de la valeur économique (Pisano, 1994; Powell, 1998; Amin et Cohendet, 2000 ; Lacetera, 2001; The Economist, 2003).

Dans les secteurs d'activité des EDB, où la science constitue le principal moteur de développement, le conseil d'administration s'adjoint généralement un conseil scientifique (Barrow, 2001; Catherine, Corolleur et Mangematin, 2003c) composé d'experts de calibre international (Bartholomey, 1997) pour l'informer

Revue internationale P.M.E., vol. 18, nº 2, 2005

(C) 2005 - Presses de l'Université du Québec

Édifice Le Delta I, 2875, boul. Laurier, bureau 450, Sainte-Foy, Québec G1V 2M2 • Tél.: (418) 657-4399 - www.puq.ca

Tiré de: Revue internationale P.M.E., vol. 18, n², sous la direction de Louis Raymond • PME1802N

Tous droits de reproduction, de traduction et d'adaptation réservés 
des nouvelles pratiques technologiques, l'assister dans ses choix stratégiques et le guider sur des questions d'éthique. En plus de ses activités dédiées à l'interne, le conseil d'administration en tant qu'interface avec l'environnement doit composer avec l'ensemble des parties prenantes (stakeholders) sur l'organisation dont il a la gouverne. On reconnait ici les actionnaires, les partenaires d'alliances (Lacetera, 2001), les associations et corporations professionnelles, divers groupes d'intérêts et, bien sûr, l'appareil gouvernemental qui résulte du contexte dans ses dimensions économique, politique, légale et sociétale. Il s'agit ici d'intégrer à la dynamique de gouvernance, l'inscription des entreprises dans un système de régulation territoriale (Gilly et Pecqueur, 1995) marqué par les proximités organisationnelle et institutionnelle des acteurs locaux entre eux. Pour circonscrire l'activité de gouvernance dans les entreprises de la grappe biopharmaceutique étudiée, nous proposons donc de l'associer à un ensemble de systèmes de surveillance et d'activités de conseil destiné à l'exécution d'une stratégie dans une organisation porteuse de valeur économique et de légitimité sociale et locale.

En résumé, dans une grappe industrielle de compétences, nous proposons de considérer que le modèle compétitif d'une entreprise se caractérise par l'exploitation d'un savoir-faire distinctif traduit dans un noyau d'habiletés reconnu par les acteurs locaux et étrangers. De façon critique, la capacité d'interaction en réseau de la firme détermine son potentiel de création de valeur. Interaction qui doit chercher à valoriser l'exploitation des quatre dynamiques définies précédemment: la capitalisation des savoirs, l'optimisation des coûts, la double logique de concurrence et de coopération et la gouvernance (figure 1).

\section{TABLEAU 1}

\section{Dimensions des quatre dynamiques territoriales d'une grappe industrielle}

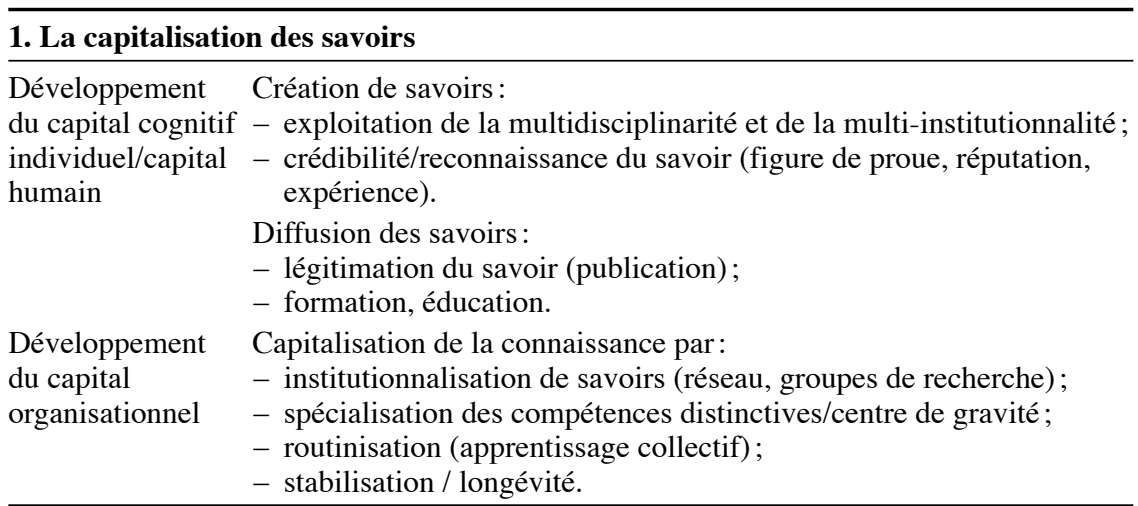


TABLEAU 1

Dimensions des quatre dynamiques territoriales d'une grappe industrielle (suite)

\begin{tabular}{ll}
\hline 2. L'optimisation des coûts \\
\hline & Ressources (ressources, compétences, capacités dynamiques) \\
\hline Activation & - humaines, \\
des ressources & - matérielles (infrastructures, équipements, stocks), \\
& - technologiques, \\
& - informationnelles, \\
& - financières, \\
& - organisationnelles (routines), \\
& - légales (subventions, lois, protections, règlements). \\
& - proximité géographique, \\
Accessibilité & - proximité économique (coût de la vie), \\
des ressources & - proximité organisationnelle (culture, structure, technique, etc.). \\
\hline
\end{tabular}

\begin{tabular}{lll}
\hline 3. La coopération/concurrence & \\
\hline Situation & Coopération & Concurrence \\
Attitude & Recherche de synergies & Accès à des ressources clés \\
& $\begin{array}{l}\text { Attitude et comportement } \\
\text { de coopération }\end{array}$ & $\begin{array}{l}\text { Attitude de domination et } \\
\text { comportement de compétition }\end{array}$ \\
Moteur & $\begin{array}{l}\text { Construction de complémentarités } \\
\text { de ressources distinctives }\end{array}$ & $\begin{array}{l}\text { Compétition pour l'accès } \\
\text { à des ressources similaires }\end{array}$ \\
Conditions & $\begin{array}{l}\text { Condition de transparence } \\
\text { Proximité culturelle }\end{array}$ & $\begin{array}{l}\text { Taille critique de l'entreprise } \\
\text { Capacité financière }\end{array}$ \\
& Maillages locaux & Barrières à l'entrée \\
Développement & $\begin{array}{l}\text { Confiance (capital social) } \\
\text { Engagement }\end{array}$ & $\begin{array}{l}\text { Rapport d'autorité } \\
\text { Rapport marchand }\end{array}$ \\
& $\begin{array}{l}\text { Alliances et collaborations } \\
\text { (réciprocité) }\end{array}$ & $\begin{array}{l}\text { Rapport de force } \\
\text { (fusion/acquisition) }\end{array}$ \\
& $\begin{array}{l}\text { Autonomie, croissance, } \\
\text { stabilité de l'entreprise. } \\
\text { Finalité }\end{array}$ & $\begin{array}{l}\text { Autonomie, croissance, } \\
\text { (capitalisation) }\end{array}$ \\
& stabilité de l'entreprise. \\
& & \\
\hline
\end{tabular}

Revue internationale P.M.E., vol. 18, nº 2, 2005

(C) 2005 - Presses de l'Université du Québec

Édifice Le Delta I, 2875, boul. Laurier, bureau 450, Sainte-Foy, Québec G1V 2M2 - Tél.: (418) 657-4399 - www.puq.ca

Tiré de: Revue internationale P.M.E., vol. 18, n², sous la direction de Louis Raymond • PME1802N

Tous droits de reproduction, de traduction et d'adaptation réservés 
4. La gouvernance (pouvoir de légitimation)

\section{Gouvernance organisationnelle}

La façon dont le pouvoir ultime s'exerce sur une entité organisationnelle:

- s'assure de la définition des objectifs stratégiques et de l'atteinte de la performance;

- contrôle la structure de management (intégrité, réputation, imputabilité);

- compose avec les parties prenantes.

\section{Corps constituan STRUCTURE}

Conseil d'administration Conseil scientifique

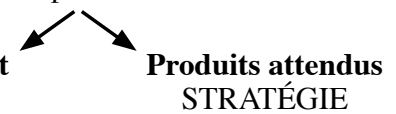

STRATÉGIE

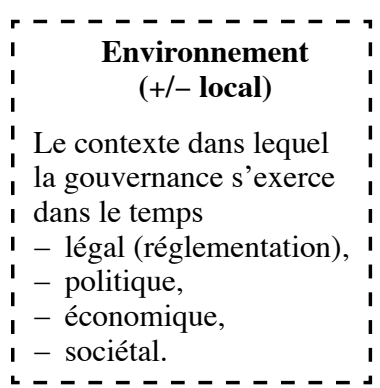

Mission, vision, positionnement.

Financement, investissement.

Création de valeur, capitalisation financière

Stabilité (âge, longévité).

FIGURE 1

La grappe biopharmaceutique (ou encore le système industriel territorial de création de valeur en santé humaine)

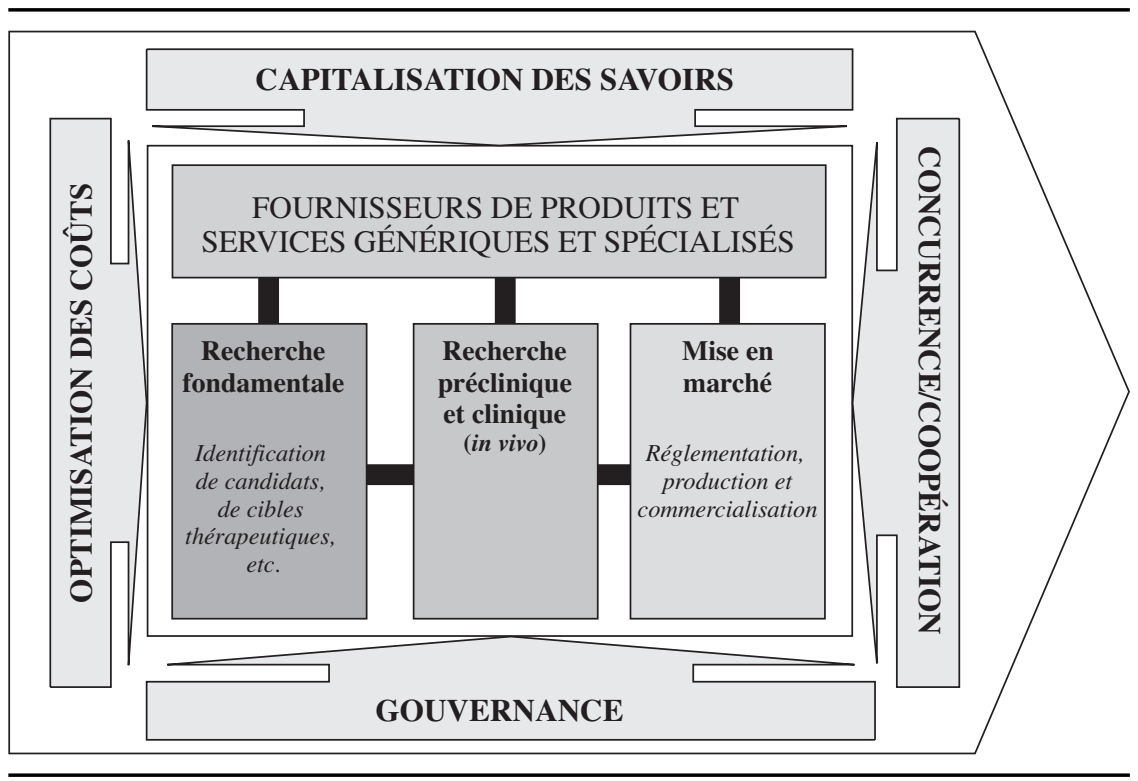

Revue internationale P.M.E., vol. 18, nº 2, 2005

(C) 2005 - Presses de l'Université du Québec

Édifice Le Delta I, 2875, boul. Laurier, bureau 450, Sainte-Foy, Québec G1V 2M2 • Tél.: (418) 657-4399 - www.puq.ca

Tiré de: Revue internationale P.M.E., vol. 18, n 2, sous la direction de Louis Raymond • PME1802N

Tous droits de reproduction, de traduction et d'adaptation réservés 


\section{Considérations méthodologiques}

\section{1. Échantillon et méthode d'exploration}

Notre objet empirique d'analyse, la grappe biopharmaceutique de la région de Montréal, représente la principale grappe industrielle d'entreprises valorisant les biotechnologies au Québec avec plus de $73 \%$ de la population totale estimée des entreprises industrielles des bio-industries québécoises parmi lesquelles $60 \%$ exercent leurs activités dans le secteur de la santé humaine. Elle est aussi l'une des trois plus importantes grappes du Canada, avec Toronto et Vancouver. Pour l'analyser, à partir du cadre théorique proposé jusqu'ici, nous utiliserons quelques-unes de nos données tirées d'une vaste série d'enquêtes par entretiens semi-directifs menées récemment auprès de l'ensemble des entreprises des bioindustries du Québec (Desmarteau, Niosi et Saives, 2002). La population estimée des entreprises de la grappe biopharmaceutique montréalaise est décrite dans le tableau 2. A priori, on dénombrerait environ $42 \%$ de firmes œuvrant en recherche fondamentale, $15 \%$ en recherche ou développement clinique, $23 \%$ en commercialisation de produits pharmaceutiques et $19 \%$ des firmes seraient des fournisseurs de produits et services spécialisés. Dans cette population, 45 entreprises industrielles des bio-industries spécialisées dans le secteur de la santé humaine dans la région de Montréal sont ici étudiées (soit 36\% de la population estimée). Il s'agit de l'ensemble des entreprises ayant accepté de répondre à nos questions dans la perspective exhaustive de notre enquête et non d'un échantillon stratifié.

TABLEAU 2

Population estimée des entreprises de la grappe industrielle de biotechnologie de la région de Montréal, 2000

\begin{tabular}{l|c|c|c}
\hline & $\begin{array}{l}\text { Nombre total } \\
\text { d'entreprises }\end{array}$ & $\begin{array}{l}\text { Nombre total } \\
\text { d'employés en } \\
\text { sciences de la vie }\end{array}$ & $\begin{array}{l}\text { Nombre total } \\
\text { d'employés } \\
\text { au Québec }\end{array}$ \\
\hline \begin{tabular}{l|c|c} 
Recherche fondamentale \\
Recherche clinique ou
\end{tabular} & $42 \%$ & $23 \%$ & $16 \%$ \\
développement technique & $15 \%$ & $17 \%$ & $21 \%$ \\
Mise en marché & $23 \%$ & $50 \%$ & $57 \%$ \\
Fournisseurs spécialisés & $19 \%$ & $9 \%$ & $6 \%$ \\
\hline
\end{tabular}

Trente-deux variables nominales ont été utilisées pour documenter les comportements de ces entreprises relativement aux quatre dynamiques territoriales décrites précédemment. Ces variables sont distribuées selon les quatre dynamiques territoriales dans la quatrième colonne de la figure $3 b$ (p. 103). Nous les expliciterons également au fur et à mesure de la description des résultats obtenus dans les

Revue internationale P.M.E., vol. 18, n 2, 2005

(C) 2005 - Presses de l'Université du Québec

Édifice Le Delta I, 2875, boul. Laurier, bureau 450, Sainte-Foy, Québec G1V 2M2 - Tél.: (418) 657-4399 - www.puq.ca

Tiré de: Revue internationale P.M.E., vol. 18, n², sous la direction de Louis Raymond • PME1802N

Tous droits de reproduction, de traduction et d'adaptation réservés 
tableaux statistiques descriptifs (numérotés 3 à 9) qui suivent. Une analyse multifactorielle en correspondances multiples (AFCM) et une classification ascendante hiérarchique $(\mathrm{CAH})$ de ces données permettent de cerner qualitativement les différents types d'acteurs présents dans la grappe et d'identifier les parangons au barycentre de ces classes d'acteurs ou encore les idéaux-types d'entreprises dont la description des comportements aura une vertu pédagogique explicative. Une analyse statistique descriptive plus précise des groupes d'entreprises relevés permet en effet par la suite de caractériser quatre modes d'ancrage territoriaux qui, en combinant différents moteurs stratégiques parmi les moteurs des quatre dynamiques territoriales présentées précédemment, façonnent la construction territoriale spécifique de l'industrie biopharmaceutique montréalaise.

\subsection{Analyse typologique des entreprises de la grappe biopharmaceutique de la région de Montréal}

La carte factorielle obtenue selon les axes factoriels 1 et 2 de l'AFCM ${ }^{12}$ est présentée à la figure 2. Elle oppose sur l'axe horizontal des entreprises n'établissant aucune alliance locale ou internationale et soumises à la compétition pour l'accès à la main-d'œuvre à des entreprises capitalisant activement le savoir dans des réseaux de relations partenariales avec les universités et d'autres entreprises, avec l'objectif de construire des actifs scientifiques et technologiques complémentaires compétitifs (axe 1). L'axe vertical (axe 2) de cette carte représente au sud des entreprises de plus petite taille bénéficiant, même difficilement, de capitalrisque pour construire une capacité d'absorption de savoirs au sein d'une plateforme technologique distinctive et d'un stock de savoirs protégé par des brevets. À l'opposé se trouvent des entreprises essentiellement caractérisées par leur taille supérieure à la moyenne.

L'étude de la carte factorielle (figure 2) et surtout l'examen de l'analyse de la $\mathrm{CAH}^{13}$ et du dendrogramme de la classification nous amènent à distinguer quatre groupes d'acteurs décrits dans le tableau 3.

- Un premier groupe (classe 1: 42,2\% des répondants) comprend un ensemble de petites et jeunes entreprises qui peuvent être qualifiées d'«entreprises dédiées de biotechnologie (EDB) "maillées" », car elles fonctionnent en réseau d'invention et d'innovation biotechnologique.

12. Pourcentage d'inertie de l'axe $1(16,1 \%)$ et de l'axe $2(8,1 \%)$. Pourcentage cumulé des cinq premiers axes $(44 \%)$.

13. Quotient (inertie inter/inertie totale) après itération: 46,7\% (idéalement, ce rapport tend vers 1). Deux jeunes entreprises de design de recherche clinique isolées ont été associées à la classe 3.1 des entreprises de recherche clinique et une entreprise dont les données, pour la plupart confidentielles, sont inutilisables a été exclue de l'analyse.

Revue internationale P.M.E., vol. 18, nº 2, 2005

(C) 2005 - Presses de l'Université du Québec

Édifice Le Delta I, 2875, boul. Laurier, bureau 450, Sainte-Foy, Québec G1V 2M2 • Tél.: (418) 657-4399 - www.puq.ca

Tiré de: Revue internationale P.M.E., vol. 18, $\mathrm{n}^{\circ} 2$, sous la direction de Louis Raymond PME1802N

Tous droits de reproduction, de traduction et d'adaptation réservés 


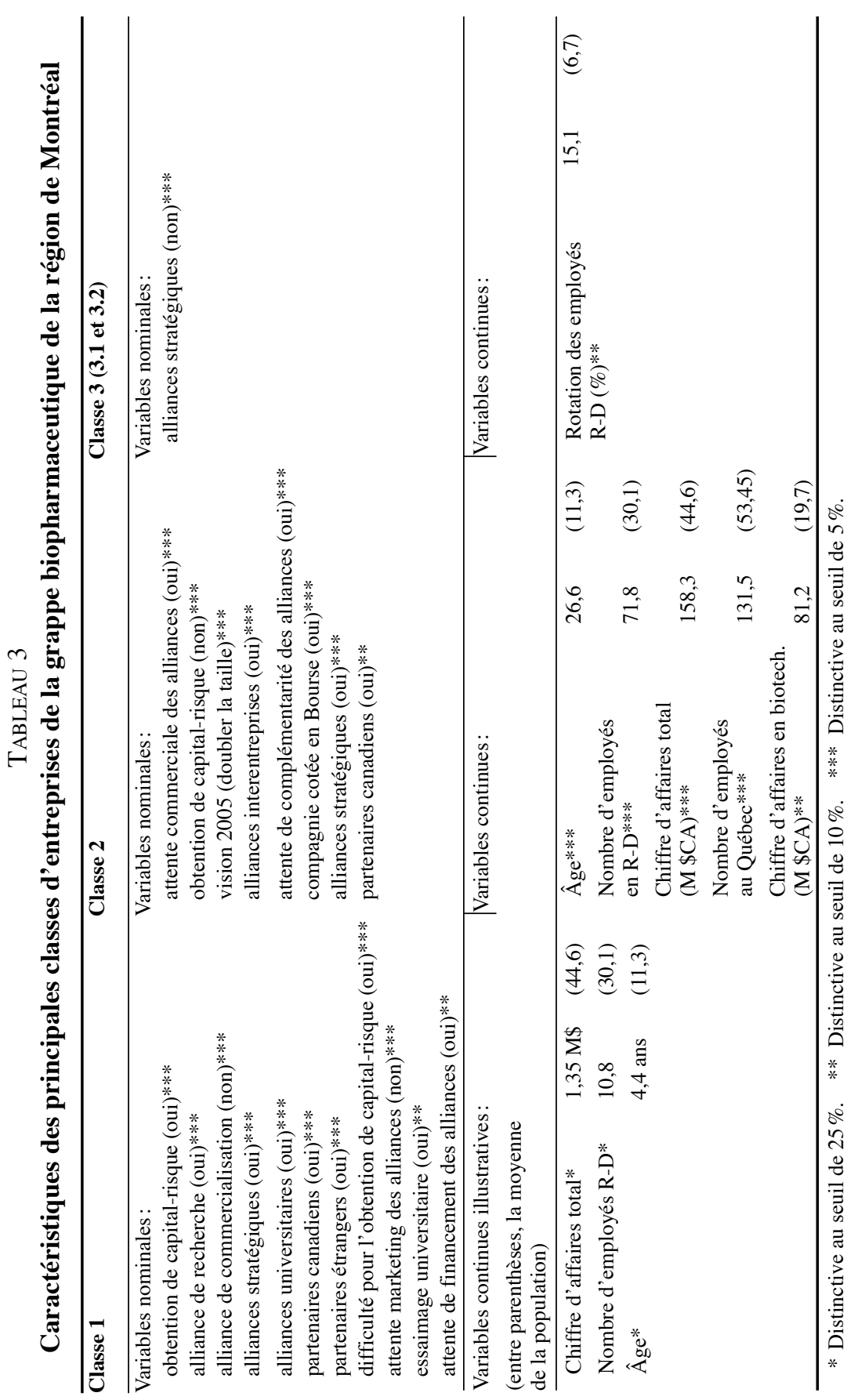

Revue internationale P.M.E., vol. 18, $\mathrm{n}^{\circ}$ 2, 2005 
FIGURE 2

\section{Typologie des entreprises de la grappe biopharmaceutique de la région de Montréal}

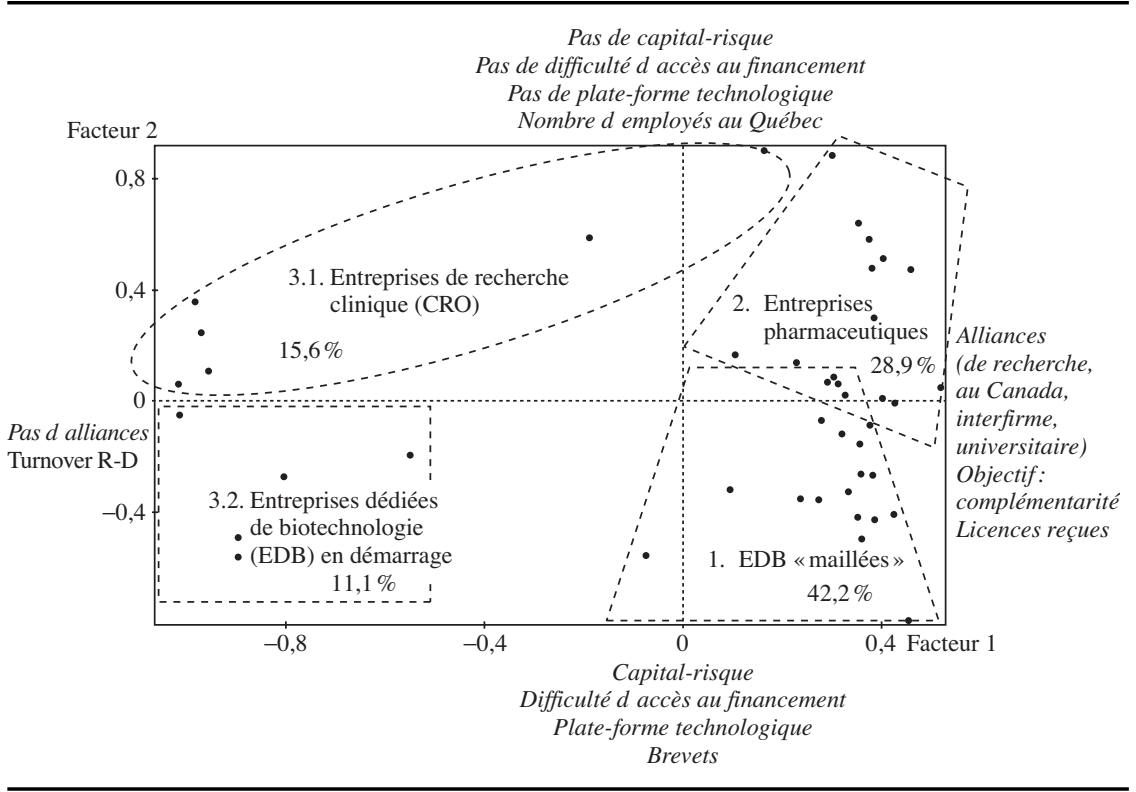

- Un deuxième groupe (classe 2: 28,9\% des entreprises) rassemble «des entreprises pharmaceutiques » de plus grande taille, caractérisées par des attentes partenariales d'abord commerciales.

- Un troisième groupe apparaît hétérogène (classe 3) sur la carte factorielle (figure 2 et dendrogramme en annexe) et on y distingue: un ensemble (sous-classe 3.1: 15,6\% des firmes) d'individus constitué «des entreprises (ou organisations) de recherche clinique» $(\mathrm{CRO})$, dont la compétence distinctive réside dans la gestion des essais cliniques. Ces entreprises sont caractérisées par un accès très concurrencé à la maind'œuvre qualifiée. On y trouve également un ensemble (sous-classe 3.2 : $11,1 \%$ des firmes) constitué des «entreprises dédiées (EDB) d'innovation biotechnologique en phase de démarrage» qui n'ont pas encore établi les bases de réseaux d'innovation et d'industrialisation biotechnologique.

Ces quatre types d'entreprises s'articulent entre eux pour constituer la grappe biopharmaceutique dont nous avons illustré les trois séquences d'activités sur la figure $1 \mathrm{au}$ début de cet article. Les classes 1 et 3.2 prennent en charge la recherche fondamentale. La classe 3.1 regroupe les activités de recherche clinique 
(en particulier les phases II, III et IV de la recherche clinique). La classe 2 composée des entreprises pharmaceutiques intègre les activités finales de validation clinique (phases IV et approbation réglementaire), de marketing, de production et de distribution.

\section{L'expression des dynamiques territoriales stratégiques au sein de la grappe biopharmaceutique de Montréal}

Partant des quatre types d'entreprises observés dans la région de Montréal, nous proposons de contextualiser maintenant les quatre dynamiques territoriales de création de valeur dans une grappe de compétences en identifiant les moteurs stratégiques activés par ces entreprises.

\subsection{La capitalisation des savoirs dans la grappe biopharmaceutique montréalaise}

Comment s'effectue la capitalisation des savoirs, c'est-à-dire la création, la diffusion et l'institutionnalisation des savoirs dans les différents types d'entreprises de la grappe biopharmaceutique de la région de Montréal?

À la lecture des indicateurs présentés dans le tableau 4, il apparaît que la création de savoirs se réalise en étroite collaboration avec les universités pour les entreprises dédiées de biotechnologies maillées (classe 1). Plus des deux tiers d'entre elles mènent des alliances de R-D avec les universités qu'elles considèrent comme un facteur de localisation très important. Cette création de savoirs s'opère en réseau mais sur la base de capacités propres d'absorption de savoirs établies et complémentaires (Powell, 1998) puisque près de 74 \% des EDB maillées possèdent et développent des capacités de recherche internes permanentes. Les entreprises pharmaceutiques (classe 2) privilégient aussi les alliances interentreprises pour capter les savoirs et alimenter les pipelines de leur développement technique.

En dehors du vecteur d'information que constituent les alliances (Baum, Calabrese et Silverman, 2000), la diffusion des savoirs s'effectue manifestement dans la région de Montréal par un phénomène récent, accéléré depuis 1998, d'essaimage universitaire dans le cas des entreprises dédiées de biotechnologies en phase de démarrage (classe 3.2) ou non (classe 1). Dans le cas des entreprises pharmaceutiques, cette diffusion des savoirs se fait indirectement par l'acquisition de licences technologiques auprès d'entreprises ou d'universités partenaires.

Revue internationale P.M.E., vol. 18, nº 2, 2005

(c) 2005 - Presses de l'Université du Québec

Édifice Le Delta I, 2875, boul. Laurier, bureau 450, Sainte-Foy, Québec G1V 2M2 • Tél.: (418) 657-4399 - www.puq.ca

Tiré de: Revue internationale P.M.E., vol. 18, $\mathrm{n}^{\circ} 2$, sous la direction de Louis Raymond • PME1802N

Tous droits de reproduction, de traduction et d'adaptation réservés 


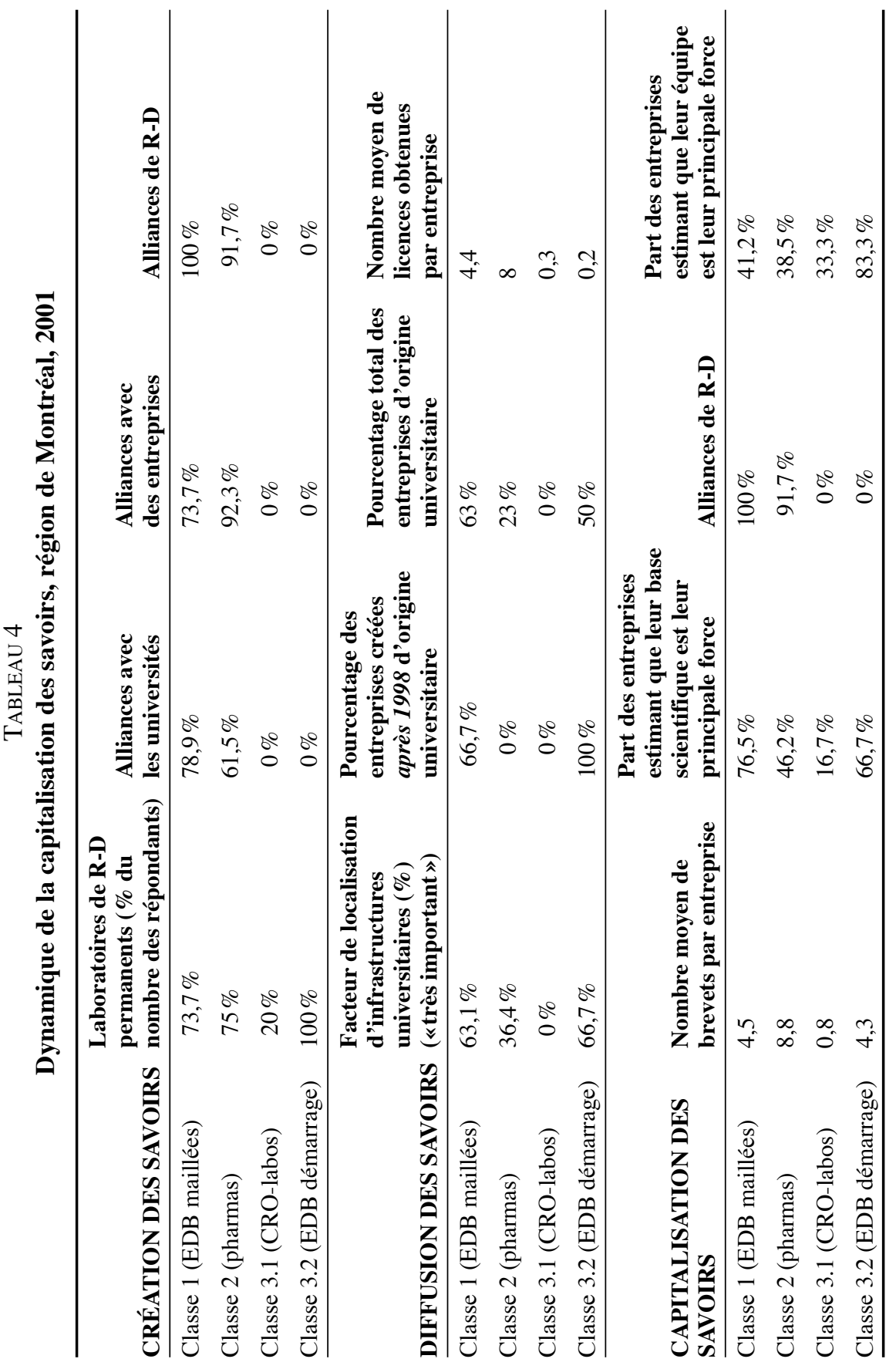

Revue internationale P.M.E., vol. 18, $\mathrm{n}^{\circ}$ 2, 2005

(C) 2005 - Presses de l'Université du Québec

Édifice Le Delta I, 2875, boul. Laurier, bureau 450, Sainte-Foy, Québec G1V 2M2 • Tél.: (418) 657-4399 - www.puq.ca

Tiré de: Revue internationale P.M.E., vol. 18, n², sous la direction de Louis Raymond • PME1802N

Tous droits de reproduction, de traduction et d'adaptation réservés 
Enfin, la capitalisation des savoirs s'opère différemment selon les entreprises: pour les EDB en démarrage (classe 3.2), le savoir est capitalisé en interne dans des systèmes de connaissances scientifiques de pointe, reconnus dans des brevets et/ou entretenus dans une capacité d'apprentissage incarnée dans des équipes scientifiques de pointe. Les EDB maillées créant du savoir en réseau (classe 1) l'institutionnalisent dans des routines collectives d'innovation et de perfectionnement continu de leurs plates-formes technologiques considérées comme la source principale de leur avantage concurrentiel, leur conférant une avance technologique (lead time) protégée par des brevets. Enfin, les entreprises pharmaceutiques (classe 2) paraissent exercer le leadership dans la capitalisation des savoirs en multipliant les brevets d'invention, d'une part, et en captant, d'autre part, au sein de réseaux institutionnalisés d'alliances de R-D («consortium» de Baumol, 2002, p. 25), les droits d'exploitation de licences technologiques.

Ainsi, les moteurs montréalais de la création et de la diffusion des savoirs sont arrimés aux universités et centres de recherche montréalais, d'une part, et à un tissu industriel dense et complémentaire, d'autre part. Les EDB en démarrage (classe 3.2) apparaissent plutôt comme des entreprises d'invention raffinant un savoir scientifique de pointe et développant d'abord un capital humain et organisationnel interne basé sur une capacité d'absorption et de R-D fondamentale. Les EDB «en réseau» ou «maillées» (classe 1) s'apparentent à des entreprises d'invention et d'innovation routinisant leurs compétences dans des plates-formes technologiques reconnues. Enfin, les entreprises pharmaceutiques (classe 2) participent à la fois du processus d'innovation et d'industrialisation de l'innovation en accumulant, d'une part, comme les précédentes, des savoirs fondamentaux et, d'autre part, en développant les innovations et en standardisant les technologies captées dans les licences de leurs partenaires.

Pour résumer les traits communs des entreprises de la grappe biopharmaceutique montréalaise, les alliances de R-D des entreprises entre elles et surtout avec les universités favorisent la dynamique de création des savoirs. La diffusion des savoirs se réalise principalement à travers des processus d'essaimage académique dans les petites entreprises (EDB des classes 1 et 3.2). Ces alliances conduisent à la capitalisation des connaissances par la construction de compétences et de capacités organisationnelles distinctives d'innovation essentiellement reconnues dans des brevets et des plates-formes technologiques avancées.

\subsection{L'optimisation des coûts dans la grappe biopharmaceutique montréalaise}

Comment s'effectue l'optimisation des coûts selon les quatre types d'entreprises de la grappe biopharmaceutique de la région de Montréal?

Revue internationale P.M.E., vol. 18, $\mathrm{n}^{\circ}$ 2, 2005

(c) 2005 - Presses de l'Université du Québec

Édifice Le Delta I, 2875, boul. Laurier, bureau 450, Sainte-Foy, Québec G1V 2M2 • Tél.: (418) 657-4399 - www.puq.ca

Tiré de: Revue internationale P.M.E., vol. 18, $\mathrm{n}^{\circ} 2$, sous la direction de Louis Raymond • PME1802N

Tous droits de reproduction, de traduction et d'adaptation réservés 
Si une grande proportion des employés recensés à Montréal se situe dans les fonctions de «fabrication» $(25 \%)$, du fait de la présence de leaders de l'industrie pharmaceutique, $21 \%$ des effectifs des entreprises de la région de Montréal œuvrent en R-D et $13 \%$, en recherche clinique. Les entreprises de la région de Montréal sont donc obligées d'optimiser d'abord leurs coûts de recherche et de fabrication. Or, la logique d'optimisation des coûts est d'abord «fiscale» quel que soit le type d'entreprise: plus des deux tiers des entreprises de Montréal considèrent les crédits fiscaux comme un facteur de localisation très important (tableau 5). Néanmoins, les entreprises de recherche clinique (classe 3.1) activent plus particulièrement cette ressource puisque pour ces entreprises, ce taux est de $100 \%$. En d'autres mots, les avantages fiscaux, dont le crédit d'impôt fédéral et provincial à la R-D, sont un facteur clé dans l'effort de réduction des coûts de la recherche à Montréal ${ }^{14}$.

TABleau 5

Indicateurs de la logique de réduction des coûts, région de Montréal, 2001

\begin{tabular}{llll}
\hline & $\begin{array}{l}\text { Facteurs de } \\
\text { localisation : } \\
\text { avantages fiscaux } \\
\text { (très important) }\end{array}$ & $\begin{array}{l}\text { Facteurs de } \\
\text { localisation : coût de } \\
\text { la vie (très important) }\end{array}$ & $\begin{array}{l}\text { Facteurs de } \\
\text { localisation : } \\
\text { disponibilité } \\
\text { de services } \\
\text { (très important) }\end{array}$ \\
\hline $\begin{array}{l}\text { Classe 1 } \\
\text { (EDB maillées) }\end{array}$ & $78,9 \%$ & $16,7 \%$ & $23,5 \%$ \\
$\begin{array}{l}\text { Classe 2 } \\
\text { (pharmas) }\end{array}$ & $61,5 \%$ & $45,4 \%$ & $27,3 \%$ \\
$\begin{array}{l}\text { Classe 3.1 } \\
\text { (CRO-labos) }\end{array}$ & $100 \%$ & $50 \%$ & $33,3 \%$ \\
$\begin{array}{l}\text { Classe 3.2 } \\
\text { (EDB démarrage) }\end{array}$ & $66,7 \%$ & $16,6 \%$ & $33,3 \%$ \\
\hline
\end{tabular}

L'importance du facteur économique du coût de la vie est aussi à rapporter au coût de la main-d'œuvre en général, qui constitue un trait distinctif de Montréal: ici, les entreprises pharmaceutiques et de recherche clinique, consommatrices de main-d'œuvre, donnent la tonalité. Elles adoptent en effet un raisonnement d'envergure internationale. Le coût de la vie à Montréal est particulièrement attractif relativement aux grandes métropoles américaines et européennes (confirmé par KPMG, 2004).

14. «The cost of doing research here!», selon l'un de nos interlocuteurs d'une grande entreprise pharmaceutique. 
Enfin, il est à noter que les jeunes entreprises (classe 3.1 et 3.2) citent comme un facteur principal de leur localisation la combinaison d'une infrastructure publique et d'un secteur tertiaire développé, car ces infrastructures permettent de diminuer les frais d'exploitation (essentiellement à travers les dispositifs d'incubation) pour des entreprises en phase de démarrage.

Pour résumer, les moteurs de l'optimisation des coûts dans la grappe biopharmaceutique montréalaise résident pour tous les groupes d'entreprises dans la proximité des stratégies publiques de développement local (politique fiscale) qui soutiennent leur orientation stratégique (Bartholomey, 1997). L'accessibilité des ressources infrastructurelles (incubateurs notamment) allègent également les frais de fonctionnement des entreprises les plus récentes (classes 3.1 et 3.2). Conjugués au moteur de la proximité économique (coût de la vie), ils accroissent l'accessibilité aux ressources humaines pour les entreprises de R-D et manufacturières (classe 2 et 3.1).

\subsection{La double logique de la concurrence et de la coopération dans la région de Montréal}

Comment s'exprime la logique de concurrence montréalaise ?

Si l'obtention de capital de risque constitue le premier enjeu de concurrence pour les EDB maillées (classe 1) et les EDB en démarrage (classe 3.2), avec des taux de difficultés d'accès qui dépassent $50 \%$, l'examen du tableau 6 révèle que seule la disponibilité de ressources qualifiées est un enjeu de concurrence partagé par tous les types d'entreprises. Plus encore, les répondants des entreprises de recherche clinique (CRO, classe 3.1) affirment que la disponibilité de ressources qualifiées constitue une problématique aiguë avec un taux de rotation des employés qui dépasse $25 \%$, et ce, tout en maintenant des activités d'exportation dans plus de $80 \%$ des cas. Bref, la recherche de ressources humaines qualifiées stigmatise les CRO, dynamise les entreprises pharmaceutiques (classe 2) et s'ajoute à l'obtention de financement comme le deuxième enjeu de concurrence pour les entreprises dédiées de biotechnologies.

Comment s'exprime la logique de coopération montréalaise?

Premier trait de la coopération montréalaise: plus de $75 \%$ des entreprises, toutes catégories confondues, sont activement engagées dans des collaborations. De façon distinctive et décisive avec un taux de $100 \%$, les entreprises maillées de biotechnologie (classe 1) et les entreprises pharmaceutiques (classe 2) mènent spécifiquement des alliances (ententes contractuelles). Ces alliances visent d'abord à établir des complémentarités scientifiques et technologiques avec les universités locales, d'autres entreprises ou encore les laboratoires publics. De plus, les EDB maillées (classe 1) estiment que l'accès à du financement est un résultat notoire

Revue internationale P.M.E., vol. 18, n 2, 2005

(C) 2005 - Presses de l'Université du Québec

Édifice Le Delta I, 2875, boul. Laurier, bureau 450, Sainte-Foy, Québec G1V 2M2 • Tél.: (418) 657-4399 - www.puq.ca

Tiré de: Revue internationale P.M.E., vol. 18, n², sous la direction de Louis Raymond • PME1802N

Tous droits de reproduction, de traduction et d'adaptation réservés 
TABLEAU 6

La concurrence pour l'accès aux ressources, région de Montréal, 2001

\begin{tabular}{lllll}
\hline & $\begin{array}{l}\text { Obstacle: } \\
\text { disponibilité } \\
\text { de ressources } \\
\text { humaines } \\
\text { qualifiées }\end{array}$ & $\begin{array}{l}\text { Part des } \\
\text { Renouvellement } \\
\text { des employés } \\
\text { de R-D }\end{array}$ & $\begin{array}{l}\text { entreprises } \\
\text { ayant eu des } \\
\text { difficultés pour } \\
\text { obtenir du } \\
\text { capital-risque }\end{array}$ & $\begin{array}{l}\text { Part des } \\
\text { entreprises } \\
\text { qui exportent }\end{array}$ \\
\hline $\begin{array}{l}\text { Classe 1 } \\
\text { (EDB maillées) }\end{array}$ & $38,9 \%$ & $4,3 \%$ & $52,6 \%$ & $27,8 \%$ \\
$\begin{array}{l}\text { Classe 2 } \\
\text { (pharmas) }\end{array}$ & $46,2 \%$ & $2,8 \%$ & 0 & $50 \%$ \\
$\begin{array}{l}\text { Classe 3.1 } \\
\text { (CRO-labos) }\end{array}$ & $60 \%$ & $25,3 \%$ & 0 & $83,3 \%$ \\
$\begin{array}{l}\text { Classe 3.2 } \\
\text { (EDB démarrage) }\end{array}$ & $33 \%$ & $2,7 \%$ & $50 \%$ & $16,7 \%$ \\
\hline
\end{tabular}

TABLEAU 7

Les indicateurs de la coopération, région de Montréal, 2001

\begin{tabular}{|c|c|c|c|c|c|}
\hline Indicateur & Montréal & Classe 1 & Classe 2 & Classe 3.1 & Classe 3.2 \\
\hline $\begin{array}{l}\text { Part des entreprises menant } \\
\text { des alliances }\end{array}$ & $74,7 \%$ & $100 \%$ & $100 \%$ & $0 \%$ & 0 \\
\hline $\begin{array}{l}\text { Résultat obtenu des alliances } \\
\text { (en \% des répondants menant } \\
\text { des alliances) }\end{array}$ & & & & & \\
\hline $\begin{array}{l}\text { Accès à des expertises } \\
\text { complémentaires scientifiques } \\
\text { et technologiques }\end{array}$ & $69 \%$ & $66,7 \%$ & $84,6 \%$ & 0 & 0 \\
\hline Accès à du financement & $40,4 \%$ & $55,6 \%$ & $25 \%$ & 0 & 0 \\
\hline Accès à la commercialisation & $34 \%$ & $0 \%$ & $38,5 \%$ & 0 & 0 \\
\hline $\begin{array}{l}\text { Accélération de la vitesse } \\
\text { de l'innovation }\end{array}$ & $76,3 \%$ & $46,5 \%$ & $76,9 \%$ & 0 & 0 \\
\hline
\end{tabular}

de leurs alliances alors que les entreprises pharmaceutiques (classe 2) en retirent une accélération de la vitesse de l'innovation. Soulignons la particularité des entreprises de recherche clinique et des EDB en démarrage (tableau 7), qui n'entretiennent pas (encore) d'alliances. Ce sont donc les EDB maillées et les entreprises pharmaceutiques qui donnent la véritable couleur de la coopération à Montréal.

Pour résumer, les forts taux de compétition tant pour les ressources humaines que financières amalgamés aux résultats des alliances conclues principalement pour l'obtention de complémentarités de savoirs nous indiquent que la double 
logique de concurrence et de coopération est bien présente dans la grappe biopharmaceutique de Montréal. En d'autres mots, il existe, à partir de différences organisationnelles, une véritable émulation territoriale porteuse de capital économique et de capital social.

\subsection{La dynamique de gouvernance dans la grappe biopharmaceutique montréalaise}

Quelle est la dynamique de gouvernance en place?

Pour décrire les traits caractéristiques des entreprises objets de gouvernance en santé humaine, alors que l'âge moyen des entreprises, tous secteurs bioindustriels confondus à Montréal est de 11 ans, le tableau 8 nous révèle qu'il est de 4,4 ans pour les EDB maillées (classe 1), de 3,75 ans pour les EDB en démarrage (classe 3.2), de 8,3 ans pour les entreprises de recherche clinique (classe 3.1) et de près de 27 ans pour les entreprises pharmaceutiques (classe 2).

Globalement, malgré l'âge des entreprises pharmaceutiques, la dynamique résultante de l'ensemble des entreprises montréalaises est définitivement jeune. Parmi les autres traits spécifiques, mentionnons l'origine entrepreuneuriale et la cotation en Bourse qui se rapportent surtout aux entreprises pharmaceutiques et aux entreprises de recherche clinique, alors que l'obtention de capital de risque est d'abord le propre des EDB (classes 1 et 3.1).

Dans le même esprit que nos observations au Québec (Desmarteau, Niosi et Saives, 2002), Catherine, Corolleur et Mangematin (2003) ont constaté dans leurs travaux sur le tissu français des entreprises de biotechnologies la spécificité de la structure de gouvernance de ces entreprises, articulée entre un conseil d'administration et un conseil scientifique et mesuré son renforcement avec l'augmentation du risque perçu par les investisseurs. Dans le cas de la dynamique de gouvernance propre aux EDB montréalaises, le tableau 9 révèle que les membres externes de leurs conseils d'administration proviennent majoritairement (à 63\%) du Québec. Quant à l'origine des membres externes des conseils scientifiques, plus de la moitié proviennent de l'extérieur du Québec dont $43 \%$ de la scène internationale. L'analyse de la dynamique des corps constituants de gouvernance des EDB montréalaises étudiées met ainsi en évidence des systèmes de surveillance et d'activités conseils qui confèrent une légitimité locale aux activités managériales et une légitimité internationale (globale) aux activités scientifiques.

Pour évaluer la performance de la gouvernance de la grappe biopharmaceutique de la région de Montréal, nous avons examiné les dispositifs d'obtention de financement et l'expression de la vision entrepreneuriale locale (tableau 8). Dans notre échantillon, $87 \%$ du capital-risque obtenu jusqu'ici (en valeur) l'a été par les entreprises du secteur de la santé humaine. Les EDB (classe 1 et classe 3.2)

Revue internationale P.M.E., vol. 18, n 2, 2005

(C) 2005 - Presses de l'Université du Québec

Édifice Le Delta I, 2875, boul. Laurier, bureau 450, Sainte-Foy, Québec G1V 2M2 • Tél.: (418) 657-4399 - www.puq.ca

Tiré de: Revue internationale P.M.E., vol. 18, n², sous la direction de Louis Raymond • PME1802N

Tous droits de reproduction, de traduction et d'adaptation réservés 

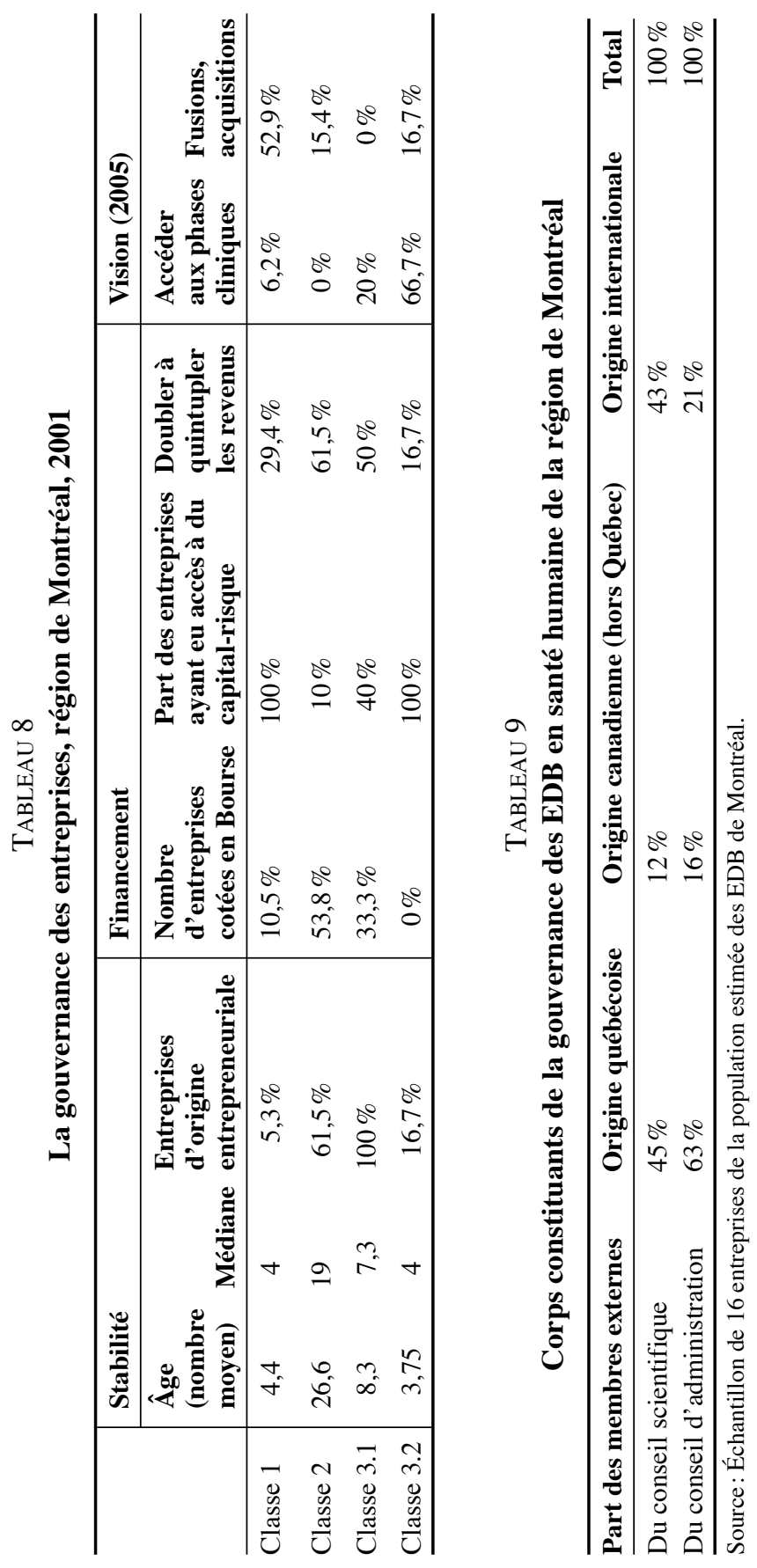

Revue internationale P.M.E., vol. 18, nº 2, 2005 
de la région de Montréal ont toutes obtenu du financement en capital-risque pour un montant moyen de 6,2 millions de dollars. Comme indices de la vitalité de la gouvernance des entreprises montréalaises, il est intéressant de constater que la majorité des répondants des EDB maillées (classe 1) prévoient participer à une fusion ou à une acquisition d'ici cinq ans, alors que, pour le même horizon, les EDB en démarrage (classe 3.1) entendent accéder aux phases supérieures des essais cliniques et les entreprises pharmaceutiques (classe 2) et les entreprises de recherche clinique, dont bon nombre sont cotées en Bourse, entendent doubler, voire quintupler leurs revenus.

En résumé, observation fut faite de la capacité de la gouvernance des firmes montréalaises d'obtenir du financement, de garantir la légitimité scientifique dans les entreprises de biotechnologie, de susciter la création de valeur et la légitimité sociale dans les entreprises pharmaceutiques et les entreprises de recherche clinique.

Néanmoins, soulignons, dans une perspective de plus long terme, quelques limites à cette dynamique. D'abord, le financement obtenu est majoritairement un financement de capital-risque institutionnel local, périodiquement qualifié d'insuffisant (IPSÉ, 2002). Ensuite, la gouvernance managériale locale ne permet pas de juger de l'attractivité des entreprises pour collecter des fonds substantiels en provenance de nouveaux investisseurs étrangers. Enfin, les entreprises cotées en Bourse ont aussi, pour certaines, précipité leur entrée sur les marchés boursiers pour capitaliser leurs activités de recherche mais souffrent, dans la conjoncture actuelle, de la difficulté de recueiller de nouveaux fonds.

\section{Conclusion}

Dans le courant des années 1990, deux secteurs industriels se sont ajoutés à la tradition industrielle pharmaceutique séculaire montréalaise pour ériger des compétences nouvelles et stratégiquement distinctives, soit les entreprises dédiées de biotechnologie et les organisations de recherche à contrat. Pour juger de l'industrialisation des innovations technologiques dans la grappe biopharmaceutique de la région de Montréal, le tableau 10 montre que plus de $40 \%$ des entreprises existantes en 2000 n'existaient pas en 1996. Ajoutons que la dimension moyenne des entreprises a augmenté pendant toute la période et que la taille médiane des plus petites entités a souvent plus que doublé entre 1996 et 1998. Ainsi, la grappe biopharmaceutique de la région de Montréal confirme son potentiel à atteindre d'ici quelques années le seuil de l'avantage compétitif dont Porter (1998) évalue le délai d'atteinte à 10 ans d'existence.

Revue internationale P.M.E., vol. 18, n 2, 2005

(C) 2005 - Presses de l'Université du Québec

Édifice Le Delta I, 2875, boul. Laurier, bureau 450, Sainte-Foy, Québec G1V 2M2 • Tél.: (418) 657-4399 - www.puq.ca

Tiré de: Revue internationale P.M.E., vol. 18, n², sous la direction de Louis Raymond • PME1802N

Tous droits de reproduction, de traduction et d'adaptation réservés 


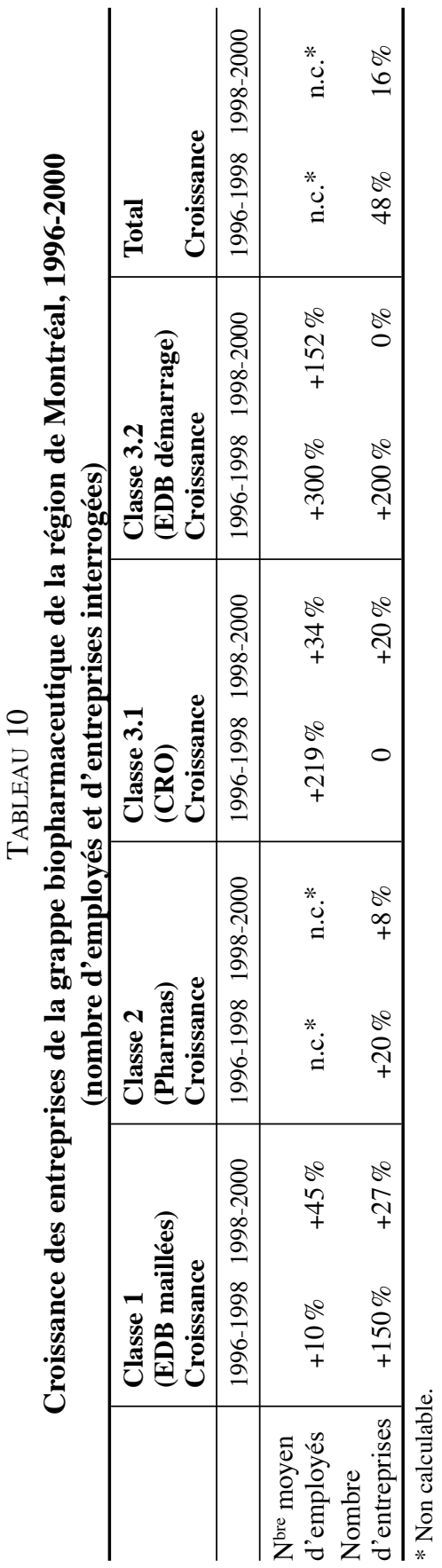

Revue internationale P.M.E., vol. 18, nº 2, 2005 
Pour comprendre l'ancrage territorial du système bio-industriel ou encore de la grappe biopharmaceutique de la région de Montréal, comme illustré dans les figures $3 \mathrm{a}$ et $3 \mathrm{~b}$, nous avons donc étudié les quatre dynamiques porteuses de valeur dans une grappe industrielle de compétences que sont la capitalisation des savoirs, l'optimisation des coûts, la double logique de concurrence et de coopération et la gouvernance. Cette analyse nous a permis de relever cinq moteurs stratégiques de création de valeur: la création de capitaux cognitifs individuels et organisationnels, l'utilisation optimale des ressources, la compétition pour les ressources, la construction de complémentarités d'action et l'organisation administrative et scientifique de gouvernance dans les entreprises du système. L'activation de ces moteurs par les différents acteurs de la scène montréalaise a favorisé le développement de facteurs d'ancrage locaux qui, en se combinant de façon idiosyncratique, façonnent quatre types d'ancrages organisationnels. Si les crédits fiscaux sont un facteur omniprésent donc non discriminant, il en est tout autrement d'activations dédiées dont les alliances de recherche avec les universités locales et de financement pour les EDB maillées, ou encore des alliances interfirmes dans des réseaux d'expertise technologique et commerciale denses pour les entreprises pharmaceutiques et, finalement, de la disponibilité de main-d'œuvre qualifiée pour les CRO.

Signalons que les EDB en démarrage présentent un profil d'ancrage similaire aux EDB maillées mais affichent la nécessité de consolider d'abord leurs propres compétences clés d'absorption des savoirs en abordant les phases de développement technique ultérieures de la recherche clinique. Elles nous rappellent aussi l'importance du paramètre temporel dans cette analyse: une étude clinique de ce jeune système d'activité, encore immature (Arora et Gambardella, 1990) et de l'évolution possible des positionnements de ces différents types d'acteurs sur leurs cycles de vie (Oliver, 2001) mériterait ainsi d'être renouvelée périodiquement, 1) pour mesurer l'impact des facteurs d'ancrage observés, notamment dans le cas de la dynamique de gouvernance entre influences locales et globales et 2) pour valider les moteurs stratégiques relevés et leur aptitude à permettre, pour les entreprises ancrées, la défense durable d'avantages concurrentiels.

Soulignons en conclusion la portée praxéologique ou encore pragmatique de la recherche présentée ici. Nous proposons de retenir la structuration du cadre exploratoire d'analyse d'un bio-cluster autour de quatre dynamiques territoriales stratégiques comme un moyen d'instrumenter les réflexions des acteurs du développement local et de l'accompagnement de la création de TPE (très petites entreprises) et PME de biotechnologie. Ces quatre dynamiques constituent un outil éclairant de description des constructions territoriales en biotechnologie et, alors que nos résultats, dans ce cas particulier (celui du biopôle de la région de Montréal), confortent les analyses menées jusqu'ici sur les bio-clusters, elles permettent aussi d'identifier les combinaisons des facteurs d'ancrage les plus

Revue internationale P.M.E., vol. 18, nº 2, 2005

(C) 2005 - Presses de l'Université du Québec

Édifice Le Delta I, 2875, boul. Laurier, bureau 450, Sainte-Foy, Québec G1V 2M2 • Tél.: (418) 657-4399 - www.puq.ca

Tiré de: Revue internationale P.M.E., vol. 18, n² 2 , sous la direction de Louis Raymond • PME1802N

Tous droits de reproduction, de traduction et d'adaptation réservés 
FIGURE 3A

Des dynamiques territoriales aux ancrages organisationnels des firmes de biotechnologie de la grappe biopharmaceutique de la région de Montréal

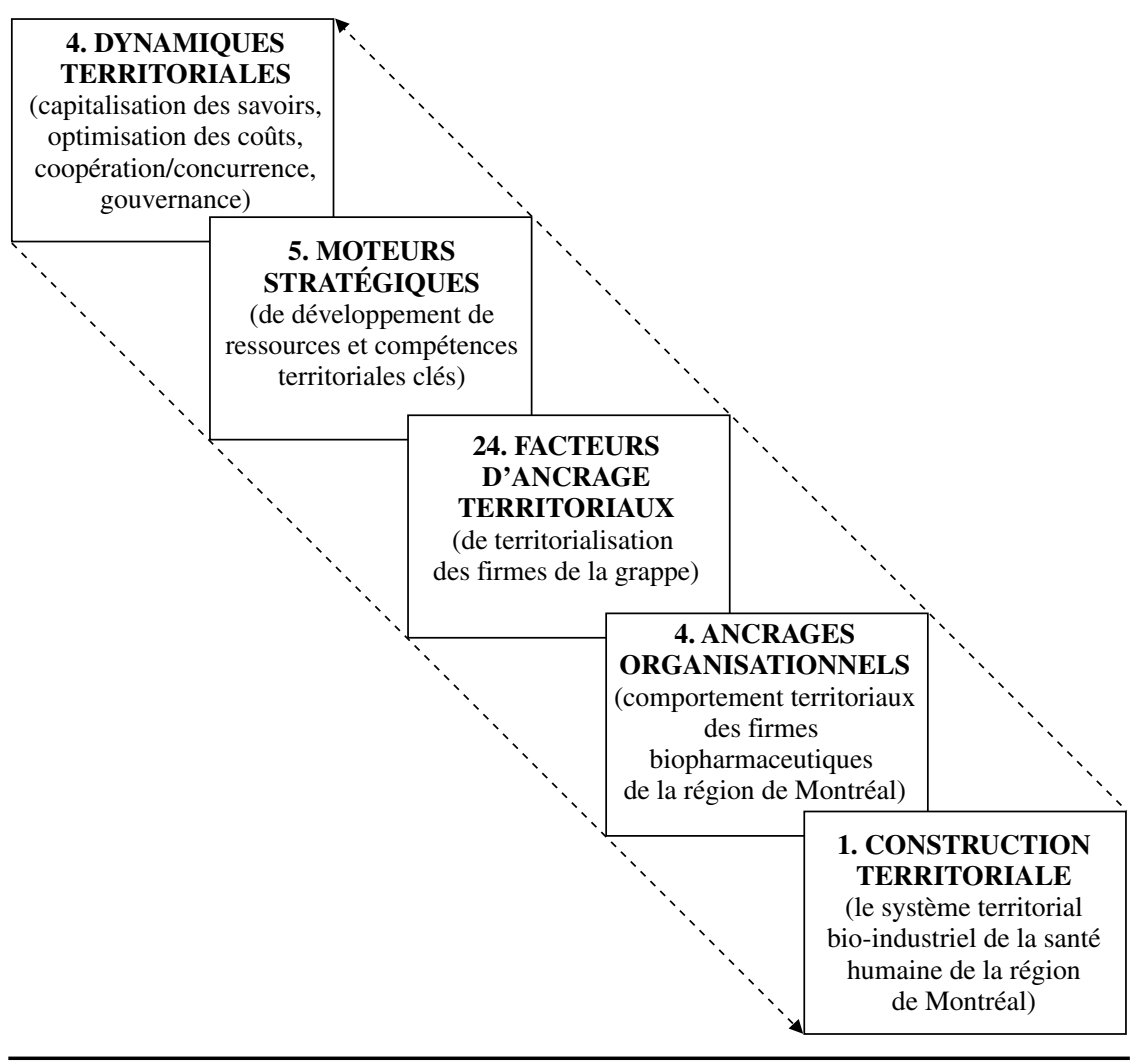

significatives selon les acteurs de la grappe du fait de l'outil d'analyse statistique typologique privilégié. En particulier, la poursuite de l'analyse des comportements des entreprises par type est révélatrice des ressources et compétences territoriales à co-construire localement dans la perspective du développement local. À ce titre, les spécificités de gestion des très petites entreprises de biotechnologie ont été récemment approfondies (Desmarteau et Saives, 2004).

Précisons enfin que l'outil à ce stade n'est qu'exploratoire et qu'il doit donc être éprouvé et validé à partir de nouvelles études de cas. À ces fins, des analyses sont en cours et à venir dans d'autres biopôles européens pour vérifier la pertinence des indicateurs choisis pour la caractérisation de chacune des dynamiques territoriales et la compréhension systémique de leur articulation entre elles. 


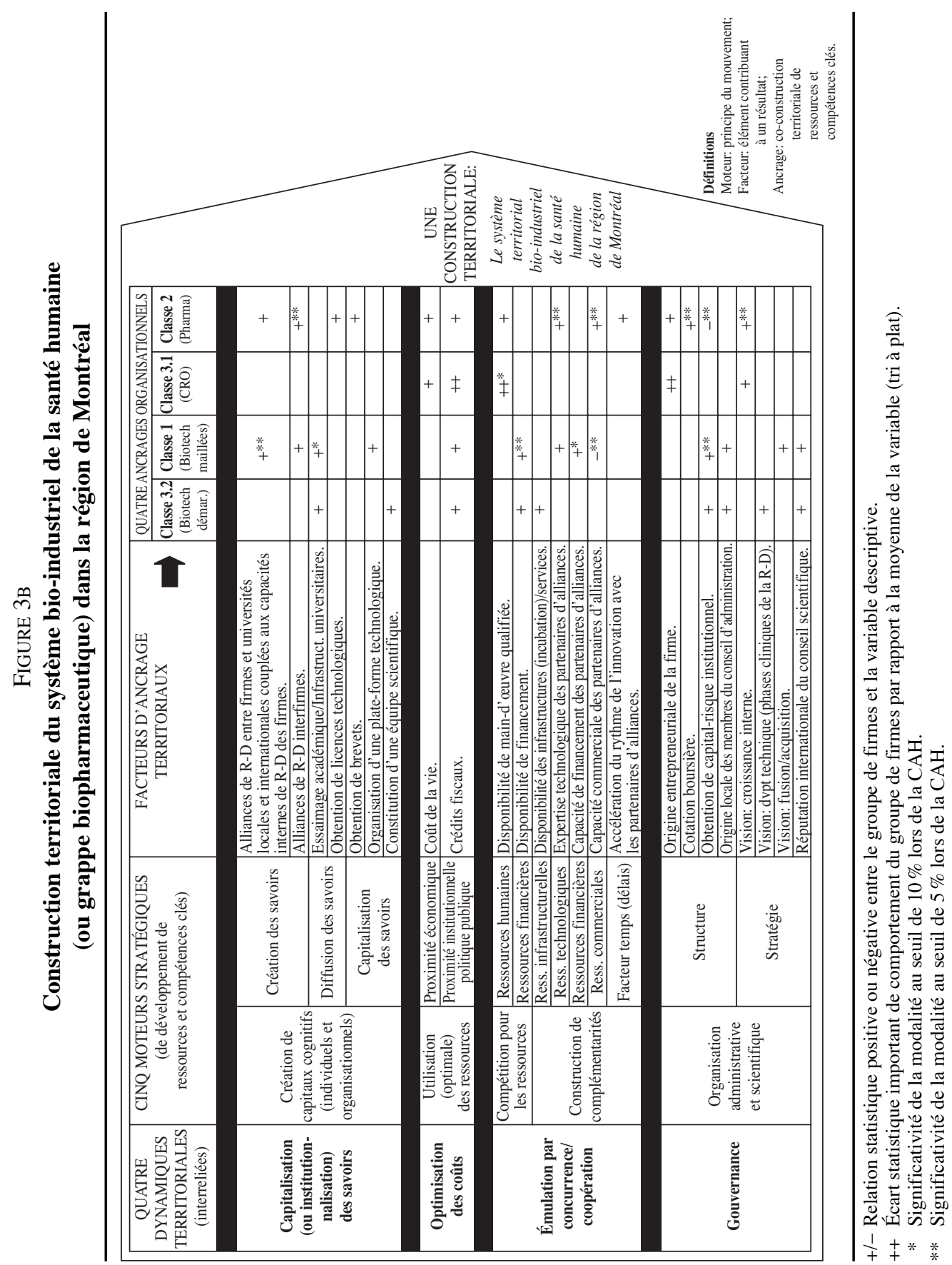

Revue internationale P.M.E., vol. 18, nº 2, 2005 


\section{Bibliographie}

ALmeIDA P. et B. Kogut (1999), «Localization of knowledge and the mobility of engineers in regional networks », Management Science, vol. 45, no 7, p. 905-917.

Amin, A. et P. CoHEndeT (2000), «Organisational learning and governance through embedded practices », Journal of Management and Governance, $\mathrm{n}^{\circ} 4$, p. 93-116.

Arora, A. et A. Gambardella (1990), «Complementarity and external linkages: the strategies of the large firms in biotechnology », The Journal of Industrial Economics, vol. 38, no 4, p. 361-379.

Aydalot, P. (1985), Économie régionale et urbaine, Paris, Economica.

BARNEY, J. (1991), «Firm resources and sustained competitive advantage», Journal of Management, vol. 17, nº 1, p. 99-120.

BARROW, C. (2001), «The role of non-executive directors in high tech SMEs », Corporate Governance, vol. 1, n 2, p. 34-36.

BARTHOLOMEY, S. (1997), «National systems of biotechnology innovation: complete interdependence in the global system», Journal of International Business Studies, $2^{\mathrm{e}}$ trimestre, p. 241-266.

Baum, J.A.C., T. Calabrese et B.S. Silverman (2000), «Don't do it alone: alliance network composition and startups' performance in Canadian biotechnology», Strategic Management Journal, vol. 21, no 3, p. 267-294.

Baumol, W. (2002), The Free-Market Innovation Machine. Analysing the Growth Miracle of Capitalism, Princeton, N.J., Princeton University Press, 318 p.

Beccattini, G. (1998), Distretti industriali e Made in Italy, Torino, Bollati Boringhieri, $195 \mathrm{p}$.

Bourdieu, P. (1986), Handbook of Theory and Research for the Sociology of Education, New York, Greenwood Press, 377 p.

Catherine, D. et F. Corolleur (2003), «Nouvelles entreprises de biotechnologies et géographie de l'innovation, des fondateurs à leur modèle d'entreprise », $R E R U, 5$, p. 785-808.

Catherine, D., F. Corolleur et R. Coronini (2003), «Les fondateurs des nouvelles entreprises de biotechnologies et leurs modèles d'entreprise », Revue internationale $P M E$, vol. 15, no 2, p. 63-92.

Catherine, D., F. Corolleur et V. Mangematin (2003), «Gouvernance par le réseau dans les PME intensives en recherche: un enjeu stratégique», XII ${ }^{\mathrm{e}}$ Conférence de l'AIMS, Tunis, 3-6 juin, 25 p.

Christensen, C.M. (1997), The Innovator's Dilemma, Boston, HBS Press.

COCKBURN, I.M. et R.M. HENDERSON (1998), «Absorptive capacity, coauthoring behavior, and the organization of research in drug discovery», The Journal of Industrial Economics, vol. 46, no 2 , p. 157-182.

Cohen, W.M. et D.A. Levinthal (1990), «Absorptive capacity: a new perspective on learning and innovation», Administrative Science Quarterly, vol. 35, p. 128-152.

Revue internationale P.M.E., vol. 18, nº 2, 2005

(C) 2005 - Presses de l'Université du Québec

Édifice Le Delta I, 2875, boul. Laurier, bureau 450, Sainte-Foy, Québec G1V 2M2 • Tél.: (418) 657-4399 - www.puq.ca

Tiré de: Revue internationale P.M.E., vol. 18, n² 2, sous la direction de Louis Raymond • PME1802N

Tous droits de reproduction, de traduction et d'adaptation réservés 
Coleman, J.S. (1988), «Social capital in the creation of human capital », American Journal of Sociology, vol. 94, supplément, p. 95-120.

Colletis, G., J.-P. Gilly et B. PeCQueur (2001), «Inscription spatiale des firmes, gouvernance des territoires et régulation d'ensemble », III Journées de la proximité, 13-14 décembre, Paris.

Corno, F., P. Reinmoeller et I. NonaKa (1999), «Knowledge creation within industrial systems », Journal of Management and Governance, $\mathrm{n}^{\circ}$ 3, p. 379-394.

Cortright, J. et H. MaYer (2002), Signs of Life: The Growth of Biotechnology Centers in the U.S., The Brookings Institution Center on Urban and Metropolitan Policy, Washington, D.C., 44 p.

DeCarolis, D.M. et D.L. DeEDs (1999), «The impact of stocks and flows of organizational knowledge on firm performance: an empirical investigation of the biotechnology industry », Strategic Management Journal, n ${ }^{\circ}$ 20, p. 953-968.

DEPRET, M.-H. et A. HAMDOUCH (2000), «Innovation networks and competitive coalitions in the pharmaceutical industry: the emergence and structures of a new industrial organization», European Journal of Economic and Social Systems, vol. 14, n 3, p. 229-270.

Desmarteau, R.H. (2000), «Le point: la valeur stratégique du capital social», ASACIFSAM, vol. 21, $\mathrm{n}^{\mathrm{o}}$ 6, p. 47-60.

Desmarteau, R.H., J. Niosi et A.-L. Saives (2002), «La biotechnologie dans la région de Montréal, analyse territoriale des entreprises», dans J. Niosi, M. Cloutier et A. Lejeune (dir.), Biotechnologie et industrie au Québec, Montréal, Éditions Transcontinental, p. 221-252.

Desmarteau, R.H. et A.-L. SAIVES (2004), «Les très petites entreprises de biotechnologies sont-elles contre-nature ? Découvrir leur identité au Québec (Canada) en explorant leur modèle d'affaires », Actes du VII ${ }^{e}$ Congrès international francophone sur l'entrepreneuriat et la PME, Montpellier, 27-29 octobre.

DIERICKX, I. et K. COOL (1989), «Asset stock accumulation and sustainability of competitive advantage », Management Science, vol. 35, n 12, p. 1504-1511.

FELDMAN, P.F. (2001), «Entrepreneurs and the formation of industrial clusters », III ${ }^{\mathrm{e}}$ Journées de la proximité, 13-14 décembre, Paris.

Fujita, M., P. Krugman P. et A. Venables (1999), The Spatial Economy: Cities, Regions and International Trade, Cambridge, Mass., MIT Press, 367 p.

Germain, A. et D. Rose (2000), Montréal, The Quest for a Metropolis, Chichester, West Sussex, R.-U., Grant, John Wiley \& Sons, 306 p.

GiLly, J.-P. et B. PeCqueur (1995), «Régulations des territoires et dynamiques institutionnelles de proximité : le cas de Toulouse et des Baronnies», dans A. Rallet et A. Torre (dir.), Économie industrielle et économie spatiale, Paris, Economica, p. 131-164.

Grant, R.M. (1996), «Toward a knowledge-based theory of the firm», Strategic Management Journal, vol. 17, hiver, p. 109-122.

Revue internationale P.M.E., vol. 18, nº 2, 2005

(C) 2005 - Presses de l'Université du Québec

Édifice Le Delta I, 2875, boul. Laurier, bureau 450, Sainte-Foy, Québec G1V 2M2 • Tél.: (418) 657-4399 - www.puq.ca

Tiré de: Revue internationale P.M.E., vol. 18, n², sous la direction de Louis Raymond • PME1802N

Tous droits de reproduction, de traduction et d'adaptation réservés 
GROUPE DE RECHERCHE SUR LES MILIEUX INNOVATEURS - GREMI (1993), Réseaux d'innovation et milieux innovateurs : un pari pour le développement régional, D. Maillat, M. Quévit et Senn (dir.), Neuchâtel, EDES.

Hofer, C.W. et D. Schendel (1978), Strategy Formulation: Analytical Concepts, St. Paul, Minn., West Publishing, 219 p.

Ingelgard, A., J. Roth, A.B. Shani et A.L. Styhre (2002), «Dynamic learning capability and actionable knowledge creation: clinical R-D in a pharmaceutical company », The Learning Organization, vol. 9, n 2, p. 65-77.

INSTITUT POUR LE PROGRÈS SOCIO-ÉCONOMIQUE - IPSÉ (2002), «Les biotechnologies au Québec: un diagnostic fondé sur huit conditions de croissance», BIOQuébec, avril.

Joffre, P. et G. KoENIG (1992), Gestion stratégique, Paris, Litec.

Julien, P.-A. et M. Marchesnay (1996), L'entrepreneuriat, Paris, Economica, 112 p.

KPMG (2004), Choix concurrentiels - Guides à l'intention des PDG sur les coûts des entreprises à l'échelle internationale, Éditions G7, site Web: <www.choixconcurrentiels.com>.

Krugman, P. (1991), «Increasing returns and economic geography», The Journal of Political Economy, vol. 99, no 3, p. 483-499.

LACETERA, N. (2001), «Corporate governance and the governance of innovation: the case of pharmaceutical industry», Journal of Management and Governance, $\mathrm{n}^{\circ} 5$, p. 29-59.

LERNER, J. (1994), «The importance of patent scope: an empirical analysis», The Rand Journal of Economics, vol. 25, $\mathrm{n}^{\circ}$ 2, p. 319-333.

Liebeskind, J., A. Oliver, L. ZuCKer et M. Brewer (1996), «Social networks, learning, and flexibility: sourcing scientific knowledge in new biotechnology firms », Organization Science, vol. 7, $\mathrm{n}^{\circ}$ 4, p. 428-443.

Marshall, A. (1890/1961), Principles of Economics, 9e édition, New York, Macmillan, $763 \mathrm{p}$.

ORGANISATION DE COOPÉRATION ET DE DÉVELOPPEMENT ÉCONOMIQUES - OCDE (1999), Boosting Innovation: The Cluster Approach, $\mathrm{n}^{\circ} 10,418 \mathrm{p}$.

ORGANISATION DE COOPÉRATION ET DE DÉVELOPPEMENT ÉCONOMIQUES - OCDE (2001a), Devolution and Globalization: Implications for Local Decision-Makers, $\mathrm{n}^{\circ} 16$, $275 \mathrm{p}$.

ORGANISATION DE COOPÉRATION ET DE DÉVELOPPEMENT ÉCONOMIQUES - OCDE (2001b), Innovative Clusters Drivers of National Innovation Systems: Enterprise, Industry and Services, $\mathrm{n}^{\circ} 17,405 \mathrm{p}$.

ORGANISATION DE COOPÉRATION ET DE DÉVELOPPEMENT ÉCONOMIQUES - OCDE (2001c), $D u$ bien-être des nations : le rôle du capital social, site Web : <www.ocde.org>.

ORGANISATION DE COOPÉRATION ET DE DÉVELOPPEMENT ÉCONOMIQUES - OCDE (2002), site Web : <http://webnet1.oecd.org/FR/document/0, FR-document-617-1-no-21-31006617---,00.html>.

Revue internationale P.M.E., vol. 18, nº 2, 2005

(C) 2005 - Presses de l'Université du Québec

Édifice Le Delta I, 2875, boul. Laurier, bureau 450, Sainte-Foy, Québec G1V 2M2 • Tél.: (418) 657-4399 - www.puq.ca

Tiré de: Revue internationale P.M.E., vol. 18, $\mathrm{n}^{\circ} 2$, sous la direction de Louis Raymond PME1802N

Tous droits de reproduction, de traduction et d'adaptation réservés 
OLIVER, A.L. (2001), «Strategic alliances and the learning life-cycle of biotechnology firms », Organization Studies, vol. 22, no 3, p. 467-489.

Parolini, C. (1999), The Value Net, Chichester, Angleterre, John Wiley \& Sons, 239 p.

Penrose, E. (1959), Theory of the Growth of the Firm, New York, John Wiley \& Sons, $271 \mathrm{p}$.

PERRIN, J.C. (1991), «Réseaux d'innovation. Milieux innovateurs développement territorial», Revue d'économie régionale et urbaine, $\mathrm{n}^{\text {os }}$ 3-4, p. 343-374.

PICARD, L. (1986), Rapport du comité consultatif au comité ministériel sur le développement de la région de Montréal, Gouvernement du Canada, Comité ministériel sur le développement de la région de Montréal, novembre, $361 \mathrm{p}$.

PISANO, G.P. (1991), «The governance of innovation: vertical integration and collaborative arrangements in the biotechnology industry », Research Policy, vol. 30, n 3, p. 237-250.

PISANO, G.P. (1994), «Knowledge, integration, and the locus of learning; an empirical analysis of process development», Strategic Management Journal, vol. 15, hiver, p. 85-100.

PorTer, M.E. (1993), L'avantage concurrentiel des nations, Paris, InterÉditions, 883 p.

PORTER, M.E. (1998), «Clusters and competition, new agendas for companies, governments, and institutions », dans On Competition, Boston, Mass., Harvard Business School Press, 485 p.

PowELL, W.W. (1998), «Learning from collaboration: knowledge and networks in the biotechnology and pharmaceutical industries», California Management Review, vol. 40 , no 3 , p. 228-240.

Powell, W.W., K.W. Koput et L. SMith-DoERR (1996), «Interorganizational collaboration and the locus of innovation: networks of learning in biotechnology », Administrative Science Quarterly, no 41, p. 116-145.

Putnam, R.D. (1993), Making Democracy Work, Civic Traditions in Italy, Princeton, N.J., Princeton University Press, 258 p.

Rallet, A. et A. Torre (1995), «Économie industrielle et économie spatiale: un état des lieux», Économie industrielle et économie spatiale, Paris, Economica, p. 3-37.

Roelandt, T.J.A. et P. Den Hertog (1999), «Cluster analysis and cluster-based policy making in OECD countries: an introduction to the theme», dans Boosting Innovation: The Cluster Approach, Organisation de coopération et de développement économiques (OCDE).

SAlman, N. et A.-L. SAIVES (2005), «Indirect networks: an intangible resource for biotechnology innovation», $R-D$ Management, vol. $35, \mathrm{n}^{\circ}$ 2, p. 203-215.

SAIVes, A.-L. (2002), Territoire et compétitivité de l'entreprise, Paris, L'Harmattan, $492 \mathrm{p}$.

Saives, A.-L., R.H. Desmarteau et D. Seni (2003), «Y a-t-il des bio-industries? Fondements et représentations», document 21-2002, Montréal, Université du Québec à Montréal, École des sciences de la gestion, Centre de recherche en gestion.

Revue internationale P.M.E., vol. 18, n 2, 2005

(C) 2005 - Presses de l'Université du Québec

Édifice Le Delta I, 2875, boul. Laurier, bureau 450, Sainte-Foy, Québec G1V 2M2 • Tél.: (418) 657-4399 - www.puq.ca

Tiré de: Revue internationale P.M.E., vol. 18, n², sous la direction de Louis Raymond • PME1802N

Tous droits de reproduction, de traduction et d'adaptation réservés 
SHAVER, J.M. et F. FlYER (2000), «Agglomeration economies, firm heterogeneity, and foreign direct investment in the United States», Strategic Management Journal, vol. $21, \mathrm{n}^{\circ} 12$, p. $1175-1193$.

SIERRA, C. (1997), «Proximité(s), interactions technologiques et territoriales: une revue », Revue d'économie industrielle, vol. 82, 4 trimestre, p. 7-38.

StUART, T. et O. SORENSON (2003), «The geography of opportunity: spatial heterogeneity in founding rates and the performance of biotechnology firms », Research Policy, $\mathrm{n}^{\circ} 32$, p. 229-253.

Stewart, G.B. (1991), The Quest for Value, New York, Harper Business, cité par P. André, A. Mersereau et R. Morissette (1998), «Valeur économique ajoutée et tableaux de bord: une combinaison stratégique», Gestion, vol. 23, n 2, p. 14-19.

SuRLEMONT, B. (2000), «Les clusters : moteurs de dynamiques entrepreneuriales », Reflets et perspectives, vol. XXXIX, no 4, p. 115-121.

THE ECONOMIST (2003), «The way we govern now», 11 janvier, p. 59-61.

Williamson, O.E. (1985), The Economic Institutions of Capitalism, New York, The Free Press, $450 \mathrm{p}$.

ZIMMERMANN, J.-B. (2001), «The firm/territory relationships in the globalisation: towards a new rationale», European Journal of Economic and Social Systems, vol. 15, no 1 , p. $57-75$. 